\title{
Analyticity of Entropy Rate of Hidden Markov Chains
}

\author{
Guangyue Han and Brian Marcus, Fellow, IEEE
}

\begin{abstract}
We prove that under mild positivity assumptions the entropy rate of a hidden Markov chain varies analytically as a function of the underlying Markov chain parameters. A general principle to determine the domain of analyticity is stated. An example is given to estimate the radius of convergence for the entropy rate. We then show that the positivity assumptions can be relaxed, and examples are given for the relaxed conditions. We study a special class of hidden Markov chains in more detail: binary hidden Markov chains with an unambiguous symbol, and we give necessary and sufficient conditions for analyticity of the entropy rate for this case. Finally, we show that under the positivity assumptions, the hidden Markov chain itself varies analytically, in a strong sense, as a function of the underlying Markov chain parameters.
\end{abstract}

Index Terms - Analyticity, entropy, entropy rate, hidden Markov chain, hidden Markov process.

\section{INTRODUCTION}

$\mathbf{F}$ OR $m, n \in \mathbb{Z}$ with $m \leq n$, we denote a sequence of symbols $y_{m}, y_{m+1}, \ldots, y_{n}$ by $y_{m}^{n}$. Consider a stationary stochastic process $Y$ with a finite set of states $\{1,2, \ldots, B\}$ and distribution $p\left(y_{m}^{n}\right)$. Denote the conditional distributions by $p\left(y_{n+1} \mid y_{m}^{n}\right)$. The entropy rate of $Y$ is defined as

$$
H(Y)=\lim _{n \rightarrow \infty}-E_{p}\left(\log \left(p\left(y_{0} \mid y_{-n}^{-1}\right)\right)\right)
$$

where $E_{p}$ denotes expectation with respect to the distribution $p$.

Let $Y$ be a stationary first-order Markov chain with

$$
\Delta(i, j)=p\left(y_{1}=j \mid y_{0}=i\right) .
$$

It is well known that

$$
H(Y)=-\sum_{i, j} p\left(y_{0}=i\right) \Delta(i, j) \log \Delta(i, j) .
$$

A hidden Markov chain $Z$ (or function of a Markov chain) is a process of the form $Z=\Phi(Y)$, where $\Phi$ is a function defined on $\{1,2, \ldots, B\}$ with values $\{1,2, \ldots, A\}$. Often, a hidden Markov chain is defined as a Markov chain observed in noise. It is well known that the two definitions are equivalent (the equivalence is typified by Example 4.1).

For a hidden Markov chain, $H(Z)$ turns out (see (2.4) below) to be the integral of a certain function defined on a simplex with respect to a measure due to Blackwell [4]. However, Blackwell's

Manuscript received July 12, 2005; revised August 8, 2006. The material in this paper was presented at the IEEE International Symposium on Information Theory, Adelaide, Australia, September 2005.

The authors are with the Department of Mathematics, University of British Columbia, Vancouver, BC V6T 1Z2, Canada (e-mail: ghan@math.ubc.ca; marcus@math.ubc.ca).

Communicated by S. A. Savari, Associate Editor for Source Coding.

Digital Object Identifier 10.1109/TIT.2006.885481 measure is somewhat complicated and the integral formula appears to be difficult to evaluate in most cases.

Recently, there has been a rebirth of interest in computing the entropy rate of a hidden Markov chain, and many approaches have been adopted to tackle this problem. For instance, some researchers have used Blackwell's measure to bound the entropy rate [20] and others introduced a variation [8] on bounds due to [3]. An efficient Monte Carlo method for computing the entropy rate of a hidden Markov chain was proposed independently by Arnold and Loeliger [2], Pfister et al. [25], and Sharma and Singh [31].

In another direction, [20], [12], [35] have studied the variation of the entropy rate as parameters of the underlying Markov chain vary. These works motivated us to consider the general question of whether the entropy rate of a hidden Markov chain is smooth, or even analytic [30], [32], as a function of the underlying parameters. Indeed, this is true under mild positivity assumptions:

Theorem 1.1: Suppose that the entries of $\Delta$ are analytically parameterized by a real variable vector $\vec{\varepsilon}$. If at $\vec{\varepsilon}=\vec{\varepsilon}_{0}$

1. for all $a \in\{1,2, \ldots, A\}$, there is at least one $j$ with $\Phi(j)=a$ such that the $j$ th column of $\Delta$ is strictly positive; and

2. every column of $\Delta$ is either all zero or strictly positive; then $H(Z)$ is a real analytic function of $\vec{\varepsilon}$ at $\vec{\varepsilon}_{0}$.

Note that this theorem holds if all the entries of $\Delta$ are positive. The more general form of our hypotheses is very important (see Example 4.1).

Real analyticity at a point is important because it means that the function can be expressed as a convergent power series in a neighborhood of the point. The power series can be used to approximate or estimate the function. For convenience of the reader, we recall some basic concepts of analyticity in Section III.

Several authors have observed that the entropy rate of a hidden Markov chain can be viewed as the top Lyapunov exponent of a random matrix product [11], [12], [10]. Results in [1], [22], [23], [27] show that under certain conditions the top Lyapunov exponent of a random matrix product varies analytically as either the underlying Markov process varies analytically or as the matrix entries vary analytically, but not both. However, when regarding the entropy rate as a Lyapunov exponent of a random matrix product, the matrix entries depend on the underlying Markov process. So, the results from Lyapunov theory do not appear to apply directly. Nevertheless, much of the main idea of our proof of Theorem 1.1 is essentially contained in Peres [23]. In contrast to Peres' proof, we do not use the language of Lyapunov exponents and we use only basic complex analysis and no functional analysis. Also, the hypotheses in [23] do not carry over to our setting. To the 
best of our knowledge, the statement and proof of Theorem 1.1 has not appeared in the literature. For analyticity of certain other statistical quantities, see also related work in the area of statistical physics in [7], [5], [15], [6].

After discussing background in Sections II and III, we prove Theorem 1.1 in Section IV. As an example, we show that the entropy rate of a hidden Markov chain obtained by observing a binary Markov chain in binary-symmetric noise, with noise parameter $\varepsilon$, is analytic at any $\varepsilon=\varepsilon_{0} \geq 0$, provided that the Markov transition probabilities are all positive.

In Section V, we infer from the proof of Theorem 1.1 a general principle to determine a domain of analyticity for the entropy rate. We apply this to the case of hidden Markov chains obtained from binary Markov chains in binary-symmetric noise to find a lower bound on the radius of convergence of a power series in $\varepsilon$ at $\varepsilon_{0}=0$. Given the recent results of [36], which compute the derivatives of all orders at $\varepsilon_{0}=0$, this gives an explicit power series for entropy rate near $\varepsilon_{0}=0$.

In Section VI, we show how to relax the conditions of Theorem 1.1 and apply this to give more examples where the entropy rate is analytic.

The entropy rate can fail to be analytic. In Section VII, we give examples and then give a complete set of necessary and sufficient conditions for analyticity in the special case of binary hidden Markov chains with an unambiguous symbol, i.e., a symbol which can be produced by only one symbol of the Markov chain.

Finally, in Section VIII, we resort to more advanced techniques to prove a stronger version, Theorem 8.1, of Theorem 1.1. This result gives a sense in which the hidden Markov chain itself varies analytically with $\vec{\varepsilon}$. The proof of this result requires some measure theory and functional analysis, along with ideas from equilibrium states [26], which are reviewed in Appendix C. Our first proof of Theorem 1.1 was derived as a consequence of Theorem 8.1. It also follows from Theorem 8.1 that, in principle, many statistical properties in addition to entropy rate vary analytically.

Most results of this paper were first announced in [9].

\section{ITERATION ON THE SIMPLEX}

Let $W$ be the simplex, comprising the vectors

$$
\left\{w=\left(w_{1}, w_{2}, \ldots, w_{B}\right) \in \mathbb{R}^{B}: w_{i} \geq 0, \sum_{i} w_{i}=1\right\}
$$

and let $W_{a}$ be all $w \in W$ with $w_{i}=0$ for $\Phi(i) \neq a$. Let $W^{\mathbb{C}}$ denote the complex version of $W$, i.e., $W^{\mathbb{C}}$ denotes the complex simplex comprising the vectors

$$
\left\{w=\left(w_{1}, w_{2}, \ldots, w_{B}\right) \in \mathbb{C}^{B}: \sum_{i} w_{i}=1\right\}
$$

and let $W_{a}^{\mathbb{C}}$ denote the complex version of $W_{a}$, i.e., $W_{a}^{\mathbb{C}}$ consists of all $w \in W^{\mathbb{C}}$ with $w_{i}=0$ for $\Phi(i) \neq a$. For $a \in A$, let $\Delta_{a}$ denote the $B \times B$ matrix such that $\Delta_{a}(i, j)=\Delta(i, j)$ for $j$ with
$\Phi(j)=a$, and $\Delta_{a}(i, j)=0$ otherwise. For $a \in A$, define the scalar-valued and vector-valued functions $r_{a}$ and $f_{a}$ on $W$ by

$$
r_{a}(w)=w \Delta_{a} \mathbf{1}
$$

and

$$
f_{a}(w)=w \Delta_{a} / r_{a}(w)
$$

Note that $f_{a}$ defines the action of the matrix $\Delta_{a}$ on the simplex $W$. For any fixed $n$ and $z_{-n}^{0}$, define

$$
x_{i}=x_{i}\left(z_{-n}^{i}\right)=p\left(y_{i}=\cdot \mid z_{i}, z_{i-1}, \ldots, z_{-n}\right)
$$

(here - represent the states of the Markov chain $Y$ ), then from Blackwell [4], $\left\{x_{i}\right\}$ satisfies the random dynamical iteration

$$
x_{i+1}=f_{z_{i+1}}\left(x_{i}\right)
$$

starting with

$$
x_{-n-1}=p\left(y_{-n-1}=\cdot\right) .
$$

We remark that Blackwell showed that

$$
H(Z)=-\int \sum_{a} r_{a}(w) \log r_{a}(w) d Q(w)
$$

where $Q$, known as Blackwell's measure, is the limiting probability distribution, as $n \rightarrow \infty$, of $\left\{x_{0}\right\}$ on $W$. However, we do not use Blackwell's measure explicitly in this paper.

Next, we consider two metrics on a compact subset $S$ of the interior of a subsimplex $W^{\prime}$ of $W$. Without loss of generality, we assume that $W^{\prime}$ consists of all points from $W$ with the last $B-k$ coordinates equal to 0 . The Euclidean metric $d_{\mathbf{E}}$ on $S$ is defined as usual, namely, for $u, v \in S$

$$
u=\left(u_{1}, u_{2}, \ldots, u_{B}\right), v=\left(v_{1}, v_{2}, \ldots, v_{B}\right) \in S
$$

we have

$$
d_{\mathbf{E}}(u, v)=\sqrt{\left(u_{1}-v_{1}\right)^{2}+\left(u_{2}-v_{2}\right)^{2}+\cdots+\left(u_{k}-v_{k}\right)^{2}} .
$$

The Hilbert metric [29] $d_{\mathbf{B}}$ on $S$ is defined as follows:

$$
d_{\mathbf{B}}(u, v)=\max _{i \neq j \leq k} \log \left(\frac{u_{i} / u_{j}}{v_{i} / v_{j}}\right) .
$$

The following result is well known (for instance, see [1]). For completeness, we give a detailed proof in Appendix A.

Proposition 2.1: $d_{\mathbf{E}}$ and $d_{\mathbf{B}}$ are equivalent (denoted by $d_{\mathbf{E}} \sim$ $d_{\mathbf{B}}$ ) on any compact subset $S$ of the interior of a subsimplex $W^{\prime}$ of $W$, i.e., there are positive constants $C_{1}<C_{2}$ such that for any two points $u, v \in S$

$$
C_{1} d_{\mathbf{B}}(u, v)<d_{\mathbf{E}}(u, v)<C_{2} d_{\mathbf{B}}(u, v) .
$$

Proposition 2.2: Assume that at $\vec{\varepsilon}_{0}, \Delta$ satisfies Conditions 1 and 2 of Theorem 1.1. Then for sufficiently large $n$ and all choices of $a_{1}, \ldots, a_{n} \in\{1,2, \ldots, A\}$ and $b \in\{1,2, \ldots, A\}$ (here $a_{1}, \ldots, a_{n}$ and $b$ denote hidden Markov symbols), the 
mapping $f_{a_{n}} \circ f_{a_{n-1}} \circ \cdots \circ f_{a_{1}}$ is a contraction mapping under the Euclidean metric on $W_{b}$.

Proof: $\hat{W}_{b}=f_{b}(W)$ is a compact subset of the interior of some subsimplex $W_{b}^{\prime}$ of $W_{b}$; this subsimplex corresponds to column indices $j$ such that $\Phi(j)=b$ and the $j$ th column is strictly positive. Therefore, one can define the Hilbert metric accordingly on $\hat{W}_{b}$. Each $f_{a}$ is a contraction mapping on each $\hat{W}_{b}$ under the Hilbert metric [29]; namely, there exists $0<\rho<$ 1 such that for any $a$ and $b$, and for any two points $u, v \in \hat{W}_{b}$,

$$
d_{\mathbf{B}}\left(f_{a}(u), f_{a}(v)\right)<\rho d_{\mathbf{B}}(u, v) .
$$

Thus, for any choices of $a_{2}, a_{3}, \ldots, a_{n}$, we have

$$
\begin{aligned}
d_{\mathbf{B}}\left(f_{a_{n}} \circ f_{a_{n-1}} \circ \cdots \circ f_{a_{2}}(u), f_{a_{n}} \circ f_{a_{n-1}} \circ\right. & \left.\cdots \circ f_{a_{2}}(v)\right) \\
& <\rho^{n-1} d_{\mathbf{B}}(u, v) .
\end{aligned}
$$

By Proposition 2.1, there exists a positive constant $C$ such that

$$
\begin{aligned}
d_{\mathbf{E}}\left(f_{a_{n}} \circ f_{a_{n-1}} \circ \cdots \circ f_{a_{2}}(u), f_{a_{n}} \circ f_{a_{n-1}} \circ \cdots \circ f_{a_{2}}(v)\right) \\
<C \rho^{n-1} d_{\mathbf{E}}(u, v) .
\end{aligned}
$$

Let $L$ be a universal Lipschitz constant for any $f_{a_{1}}: W_{b} \rightarrow W_{a_{1}}^{\prime}$ with respect to the Euclidean metric. Choose $n$ large enough such that $C \rho^{n-1}<1 / L$. So, for sufficiently large $n$, any composition of the form $f_{a_{n}} \circ \cdots \circ f_{a_{1}}$ is a Euclidean contraction on $W_{b}$.

Remark 2.3: Using a slightly modified proof, one can show that for sufficiently large $n$, any composition of the form $f_{a_{n}}$ 。 $\ldots \circ f_{a_{1}}$ is a Euclidean contraction on the whole simplex $W$.

\section{BRIEF BACKGROUND ON ANALYTICITY}

In this section, we briefly review the basics in complex analysis for the purpose of this paper. For more details, we refer to [30], [32].

A real (or complex) function of several variables is analytic at a given point if it admits a convergent Taylor series representation in a real (or complex) neighborhood of the given point. We say that it is real (or complex) analytic in a neighborhood if it is real (or complex) analytic at each point of the neighborhood.

The relationship between real and complex analytic functions is as follows: 1) Any real analytic function can be extended to a complex analytic function on some complex neighborhood. 2) Any real function obtained by restricting a complex analytic function from a complex neighborhood to a real neighborhood is a real analytic function.

The main fact regarding analytic functions used in this paper is that the uniform limit of a sequence of complex analytic functions on a fixed complex neighborhood is complex analytic. The analogous statement does not hold (in fact, fails dramatically!) for real analytic functions.

As an example of a real-valued parametrization of a matrix, consider

$$
\Delta(\varepsilon)=\left[\begin{array}{ccc}
2 \varepsilon & \varepsilon & 1-3 \varepsilon \\
\varepsilon & 1-\varepsilon-\sin (\varepsilon) & \sin (\varepsilon) \\
\varepsilon^{2} & \varepsilon^{3} & 1-\varepsilon^{2}-\varepsilon^{3}
\end{array}\right] .
$$

Denote the states of $\Delta$ by $\{1,2,3\}$ and let $\Phi(1)=\Phi(2)=$ $0, \Phi(3)=1$. Each entry of $\Delta$ is a real analytic function of $\varepsilon$ at any given point $\varepsilon=\varepsilon_{0}$. For $\varepsilon_{0}>0$ and sufficiently small, $\Delta$ is stochastic (i.e., each row sums to 1 and each entry is nonnegative) and in fact strictly positive (i.e., each entry is positive). According to Theorem 1.1, for such values of $\varepsilon_{0}$, the entropy rate of the hidden Markov chain defined by $\Delta(\varepsilon)$ and $\Phi$ is real analytic as a function of $\varepsilon$ at $\varepsilon_{0}$.

While we typically think of analytic parametrizations as having the "look" of the preceding example, there is a conceptually simpler parametrization-namely, parameterize an $n \times n$ matrix $\Delta$ by its entries themselves; if $\Delta$ is required to be stochastic, we choose the parameters to be any set of $n-1$ entries in each row (so, the real variable vector is an $n(n-1)$-tuple). Clearly, for analyticity it does not matter which entries are chosen. We call this the natural parametrization.

Suppose that $H(Z)$ is analytic with respect to this parametrization. Then, $H(Z)$ viewed as a function of any other analytic parametrization of the entries of $\Delta$ is the composition of two analytic functions and thus must be analytic. We thus have that the following two statements are equivalent.

- $H(Z)$ is analytic with respect to the natural parameterization.

- $H(Z)$ is analytic with respect to any analytic parameterization.

We shall use this implicitly throughout the paper.

\section{Proof OF THEOREM 1.1}

Notation: We rewrite $\Delta, Z, f_{a}(x), p\left(z_{0} \mid z_{-n}^{-1}\right)$ with parameter vector $\vec{\varepsilon}$ as $\Delta^{\vec{\varepsilon}}, Z^{\vec{\varepsilon}}, f_{a}^{\vec{\varepsilon}}(x)$, and $p^{\vec{\varepsilon}}\left(z_{0} \mid z_{-n}^{-1}\right)$, respectively. We use the notation $\hat{W}_{a}$ to mean $f_{a}^{\varepsilon_{0}}(W)$. Let $\Omega_{\mathbb{C}}=\Omega_{\mathbb{C}}(r)$ denote the set of points of distance at most $r$ from $\vec{\varepsilon}_{0}$ in the complex parameter space $\mathbb{C}^{m}$. Let $N_{b}=N_{b}(R)$ denote the set of all points in $W_{b}^{\mathbb{C}}$ of distance at most $R$ from $\hat{W}_{b}$.

We first prove that for some $r>0, \log p^{\vec{\varepsilon}}\left(z_{0} \mid z_{-n}^{-1}\right)$ can be extended to a complex analytic function of $\vec{\varepsilon} \in \Omega_{\mathbb{C}}(r)$ and for two symbol sequences $z_{-n_{1}}^{0}$ and $\hat{z}_{-n_{2}}^{0}$

$$
\left|\log p^{\vec{\varepsilon}}\left(z_{0} \mid z_{-n_{1}}^{-1}\right)-\log p^{\vec{\varepsilon}}\left(\hat{z}_{0} \mid \hat{z}_{-n_{2}}^{-1}\right)\right|
$$

decays exponentially fast in $n$, when $n \leq n_{1}, n_{2}$ and $z_{-n}^{0}=$ $\hat{z}_{-n}^{0}$, uniformly in $\vec{\varepsilon} \in \Omega_{\mathbb{C}}(r)$.

Note that for each $a, b, f_{a}^{\vec{\varepsilon}}(w)$ is a rational function of the entries of $\Delta^{\vec{\varepsilon}}$ and $w \in \hat{W}_{b}$. So, by viewing the real vector variables $\vec{\varepsilon}$ and $w$ as complex vector variables, we can naturally extend $f_{a}^{\vec{\varepsilon}}(w)$ to a complex-valued function of complex vector variables $\vec{\varepsilon}$ and $w$. Since $\Delta$ satisfies Conditions 1 and 2 at $\vec{\varepsilon}_{0}$, for sufficiently small $r$ and $R$, the denominator of $f_{a}^{\vec{\varepsilon}}(w)$ is nonzero for $\vec{\varepsilon}$ in $\Omega_{\mathbb{C}}(r)$ and $w$ in $N_{b}(R)$. Thus, $f_{a}^{\vec{\varepsilon}}(w)$ is a complex analytic function of $(\vec{\varepsilon}, w)$ in the neighborhood $\Omega_{\mathbb{C}}(r) \times N_{b}(R)$.

Assuming Conditions 1 and 2, we claim that $\Delta$ has an isolated (in modulus) maximum eigenvalue 1 at $\vec{\varepsilon}_{0}$. To see this, we apply Perron-Frobenius theory [29] as follows. By permuting the indices, we can express

$$
\Delta=\left[\begin{array}{ll}
U & 0 \\
V & 0
\end{array}\right]
$$


where $U$ is the submatrix corresponding to indices with positive columns. The nonzero eigenvalues of $\Delta$ are the same as the eigenvalues of $U$, which is a positive stochastic matrix. Such a matrix has isolated (in modulus) maximum eigenvalue 1.

The stationary distribution $p^{\vec{\varepsilon}}(y=\cdot)$ (the eigenvector corresponding to the maximum eigenvalue 1 ) is a rational function of the entries of $\Delta^{\vec{\varepsilon}}$, since it is a solution of the equation $v \Delta^{\vec{\varepsilon}}=v$. So, in the same way as for $f_{a}^{\vec{\varepsilon}}(w)$, we can naturally extend $p^{\vec{\varepsilon}}(y=\cdot)$ to a complex analytic function $p^{\vec{\varepsilon}}(y=\cdot)$ on $\Omega_{\mathbb{C}}$.

Extending (2.1) for each $i$, we define

$$
x_{i}^{\vec{\varepsilon}}=x_{i}^{\vec{\varepsilon}}\left(z_{-n}^{i}\right)=p^{\vec{\varepsilon}}\left(y_{i}=\cdot \mid z_{-n}^{i}\right)
$$

by iterating the following complexified random dynamical system (extending (2.2) and (2.3)):

$$
x_{i+1}^{\vec{\varepsilon}}=f_{z_{i+1}}^{\vec{\varepsilon}}\left(x_{i}^{\vec{\varepsilon}}\right)
$$

starting with

$$
x_{-n-1}^{\vec{\varepsilon}}=p^{\vec{\varepsilon}}\left(y_{-n-1}=\cdot\right) \text {. }
$$

By Proposition 2.2, for sufficiently large $n$, we can replace the set of mappings $\left\{f_{a}^{\varepsilon_{0}}\right\}$ with the set $\left\{f_{a_{n}}^{\varepsilon_{0}} \circ f_{a_{n-1}}^{\varepsilon_{0}} \circ \cdots \circ f_{a_{1}}^{\varepsilon_{0}}\right\}$ and then assume that each $f_{a}^{\varepsilon_{0}}$ is a Euclidean contraction on each $W_{b}$ with contraction coefficient $\rho<1$. Since $\hat{W}_{b}$ is compact and the definition of $\rho$-contraction is given by strict inequality, we can choose $r$ and $R$ sufficiently small such that

$f_{a}^{\vec{\varepsilon}_{\text {is }}}$ a Euclidean $\rho$-contraction on each $N_{b}(R), \quad \varepsilon \in \Omega_{\mathbb{C}}(r)$.

Further, we claim that by choosing $r$ still smaller, if necessary

$x_{i}^{\vec{\varepsilon}} \in \cup_{b} N_{b}(R)$, for all $i, n$ and all choices of $z_{-n}^{i}, \quad \varepsilon \in \Omega_{\mathbb{C}}(r)$.

To see this, fixing $\rho$ and $R$, choose $r$ so small that

$$
\left|f_{a}^{\vec{\varepsilon}}(x)-f_{a}^{\vec{\varepsilon}_{0}}(x)\right| \leq R(1-\rho), x \in \cup_{b} \hat{W}_{b}, \varepsilon \in \Omega_{\mathbb{C}}(r)
$$

and

$$
\left|p^{\vec{\varepsilon}}(\cdot)-p^{\vec{\varepsilon}_{0}}(\cdot)\right| \leq R(1-\rho), \varepsilon \in \Omega_{\mathbb{C}}(r) .
$$

Now consider the difference

$$
\begin{aligned}
x_{i+1}^{\vec{\varepsilon}}-x_{i+1}^{\vec{\varepsilon}_{0}}= & f_{z_{i+1}}^{\vec{\varepsilon}}\left(x_{i}^{\vec{\varepsilon}}\right)-f_{z_{i+1}}^{\vec{\varepsilon}_{0}}\left(x_{i}^{\vec{\varepsilon}_{0}}\right) \\
= & f_{z_{i+1}}^{\vec{\varepsilon}}\left(x_{i}^{\vec{\varepsilon}}\right)-f_{z_{i+1}}^{\vec{\varepsilon}}\left(x_{i}^{\vec{\varepsilon}_{0}}\right) \\
& +f_{z_{i+1}}^{\vec{\varepsilon}}\left(x_{i}^{\vec{\varepsilon}_{0}}\right)-f_{z_{i+1}}^{\vec{\varepsilon}_{0}}\left(x_{i}^{\vec{\varepsilon}_{0}}\right) .
\end{aligned}
$$

Then by (4.8), (4.10) and (4.11), and (4.12), for $i>-n-1$, we have

$$
\left|x_{i+1}^{\vec{\varepsilon}}-x_{i+1}^{\vec{\varepsilon}_{0}}\right| \leq \rho\left|x_{i}^{\vec{\varepsilon}}-x_{i}^{\vec{\varepsilon}_{0}}\right|+R(1-\rho)
$$

So

$$
\left|x_{i+1}^{\vec{\varepsilon}}-x_{i+1}^{\vec{\varepsilon}_{0}}\right| \leq R
$$

and thus for all $i$, we have $x_{i+1}^{\vec{\varepsilon}} \in \cup_{b} N_{b}(R)$, yielding (4.9). Each $x_{i}^{\varepsilon}$ is the composition of analytic functions on $\Omega_{\mathbb{C}}(r)$ and so is complex analytic on $\Omega_{\mathbb{C}}(r)$.

For $0 \leq n_{1}, n_{2} \leq \infty$, we say two sequences $\left\{z_{-n_{1}}^{0}\right\}$ and $\left\{\hat{z}_{-n_{2}}^{0}\right\}$ have a common tail if there exists $n \geq 0$ with $n \leq n_{1}, n_{2}$ such that $z_{i}=\hat{z}_{i},-n \leq i \leq 0$ (denoted by $\left.z_{-n_{1}}^{0} \stackrel{n}{\sim} \hat{z}_{-n_{2}}^{0}\right)$.

Let

$$
\begin{aligned}
& x_{i}^{\vec{\varepsilon}}=x_{i}^{\vec{\varepsilon}}\left(z_{-n_{1}}^{i}\right)=p^{\vec{\varepsilon}}\left(y_{i}=\cdot \mid z_{-n_{1}}^{i}\right) \\
& \hat{x}_{i}^{\vec{\varepsilon}}=\hat{x}_{i}^{\vec{\varepsilon}}\left(\hat{z}_{-n_{2}}^{i}\right)=p^{\vec{\varepsilon}}\left(y_{i}=\cdot \mid \hat{z}_{-n_{2}}^{i}\right) .
\end{aligned}
$$

Then we have

$$
\begin{aligned}
x_{i+1}^{\vec{\varepsilon}} & =f_{z_{i+1}}^{\vec{\varepsilon}}\left(x_{i}^{\vec{\varepsilon}}\right) \\
\hat{x}_{i+1}^{\vec{\varepsilon}} & =f_{\hat{z}_{i+1}}^{\vec{\varepsilon}}\left(\hat{x}_{i}^{\vec{\varepsilon}}\right) .
\end{aligned}
$$

From (4.8) and (4.9), if $z_{-n_{1}}^{0} \stackrel{n}{\sim} \hat{z}_{-n_{2}}^{0}$, then there exists a positive constant $L$ independent of $n_{1}$ and $n_{2}$ such that

$$
\left|x_{0}^{\vec{\varepsilon}}-\hat{x}_{0}^{\vec{\varepsilon}}\right| \leq L \rho^{n} \text {. }
$$

Naturally

$$
p^{\vec{\varepsilon}}\left(z_{0} \mid z_{-n}^{-1}\right)=\sum_{\left\{y_{0}: \Phi\left(y_{0}\right)=z_{0}\right\}} \sum_{y_{-1}} \Delta^{\vec{\varepsilon}}\left(y_{-1}, y_{0}\right) p^{\vec{\varepsilon}}\left(y_{-1} \mid z_{-n}^{-1}\right) .
$$

Then, there is a positive constant $L^{\prime}$, independent of $n_{1}, n_{2}$, such that

$$
\left|p^{\vec{\varepsilon}}\left(z_{0} \mid z_{-n_{1}}^{-1}\right)-p^{\vec{\varepsilon}}\left(\hat{z}_{0} \mid \hat{z}_{-n_{2}}^{-1}\right)\right| \leq L^{\prime} \rho^{n}
$$

Since $\Delta^{\vec{\varepsilon}_{0}}$ satisfies Conditions 1 and $2, p^{\vec{\varepsilon}}\left(z_{0} \mid z_{-n}^{-1}\right)$ is bounded away from 0 , uniformly in $\vec{\varepsilon} \in \Omega_{\mathbb{C}}, n$ and choices of $z_{-n}^{-1}$; thus, there is a positive constant $L^{\prime \prime}$, independent of $n_{1}, n_{2}$, such that

$$
\left|\log p^{\vec{\varepsilon}}\left(z_{0} \mid z_{-n_{1}}^{-1}\right)-\log p^{\vec{\varepsilon}}\left(\hat{z}_{0} \mid \hat{z}_{-n_{2}}^{-1}\right)\right| \leq L^{\prime \prime} \rho^{n} .
$$

Since for each $y \in\{1, \ldots, B\}, p^{\vec{\varepsilon}}(y)$ is analytic, from

$$
p^{\vec{\varepsilon}}(z)=\sum_{\Phi(y)=z} p^{\vec{\varepsilon}}(y)
$$

we deduce that $p^{\vec{\varepsilon}}(z)$ is analytic. Furthermore, since $p^{\vec{\varepsilon}}\left(z_{0} \mid z_{-n}^{-1}\right)$ is analytic on $\Omega_{\mathbb{C}}$, we conclude $p^{\vec{\varepsilon}}\left(z_{-n}^{0}\right)$ is analytic on $\Omega_{\mathbb{C}}$.

Choose $\sigma$ so that

$$
1<\sigma<1 / \rho
$$

If $r$ and $R$ are chosen sufficiently small, then

$$
\sum_{z_{0}}\left|p^{\vec{\varepsilon}}\left(z_{0} \mid z_{-n}^{-1}\right)\right| \leq \sigma, \varepsilon \in \Omega_{\mathbb{C}}(r) \text { and all sequences } z
$$

and

$$
\sum_{z_{0}}\left|p^{\vec{\varepsilon}}\left(z_{0}\right)\right| \leq \sigma, \varepsilon \in \Omega_{\mathbb{C}}(r)
$$


Then we have

$$
\begin{aligned}
& \sum_{z_{-n-1}^{0}}\left|p^{\vec{\varepsilon}}\left(z_{-n-1}^{0}\right)\right| \\
& \quad=\sum_{z_{-n-1}^{0}}\left|p^{\vec{\varepsilon}}\left(z_{-n-1}^{-1}\right) p^{\vec{\varepsilon}}\left(z_{0} \mid z_{-n-1}^{-1}\right)\right| \\
& \leq \sum_{z_{-n-1}^{-1}}\left|p^{\vec{\varepsilon}}\left(z_{-n-1}^{-1}\right)\right| \sum_{z_{0}}\left|p^{\vec{\varepsilon}}\left(z_{0} \mid z_{-n-1}^{-1}\right)\right| \\
& \leq \sigma \sum_{z_{-n}^{0}}\left|p^{\vec{\varepsilon}}\left(z_{-n}^{0}\right)\right|
\end{aligned}
$$

implying

$$
\sum_{z_{-n-1}^{0}}\left|p^{\vec{\varepsilon}}\left(z_{-n-1}^{0}\right)\right| \leq \sigma^{n+2}
$$

Let

$$
H_{n}^{\vec{\varepsilon}}(Z)=-\sum_{z_{-n}^{0}} p^{\vec{\varepsilon}}\left(z_{-n}^{0}\right) \log p^{\vec{\varepsilon}}\left(z_{0} \mid z_{-n}^{-1}\right)
$$

and

$$
\rho_{1}=\rho \delta<1
$$

then we have

$$
\begin{aligned}
& \left|H_{n+1}^{\vec{\varepsilon}}(Z)-H_{n}^{\vec{\varepsilon}}(Z)\right| \\
& =\mid \sum_{z_{-n-1}^{0}} p^{\vec{\varepsilon}}\left(z_{-n-1}^{0}\right) \log p^{\vec{\varepsilon}}\left(z_{0} \mid z_{-n-1}^{-1}\right) \\
& \quad-\sum_{z_{-n}^{0}} p^{\vec{\varepsilon}}\left(z_{-n}^{0}\right) \log p^{\vec{\varepsilon}}\left(z_{0} \mid z_{-n}^{-1}\right) \mid \\
& =\mid \sum_{z_{-n-1}^{0}} p^{\vec{\varepsilon}}\left(z_{-n-1}^{0}\right)\left(\log p^{\vec{\varepsilon}}\left(z_{0} \mid z_{-n-1}^{-1}\right)\right. \\
& \left.\quad-\log p^{\vec{\varepsilon}}\left(z_{0} \mid z_{-n}^{-1}\right)\right) \mid \leq \sigma^{2} L^{\prime \prime} \rho_{1}^{n} ;
\end{aligned}
$$

here the latter inequality follows from (4.16) and (4.19). Thus, for $m>n$

$$
\begin{aligned}
\left|H_{m}^{\vec{\varepsilon}}(Z)-H_{n}^{\vec{\varepsilon}}(Z)\right| & \leq \sigma^{2} L^{\prime \prime}\left(\rho_{1}^{n}+\cdots+\rho_{1}^{m-1}\right) \\
& \leq \frac{\sigma^{2} L^{\prime \prime} \rho_{1}^{n}}{1-\rho_{1}} .
\end{aligned}
$$

This establishes the uniform convergence of $H_{n}^{\vec{\varepsilon}}(Z)$ to a limit $H_{\infty}^{\vec{\varepsilon}}(Z)$. By Theorem 2.4.1 of [32], the uniform limit of complex analytic functions on a fixed complex neighborhood is analytic on that neighborhood, and so $H_{\infty}^{\vec{\varepsilon}}(Z)$ is analytic on $\Omega_{\mathbb{C}}$.

For real $\vec{\varepsilon}, H_{\infty}^{\vec{\varepsilon}}(Z)$ coincides with the entropy rate function $H\left(Z^{\vec{\varepsilon}}\right)$, and so Theorem 1.1 follows.

Example 4.1: Consider a binary-symmetric channel with crossover probability $\varepsilon$. Let $\left\{Y_{n}\right\}$ be the input Markov chain with the transition matrix

$$
\Pi=\left[\begin{array}{ll}
\pi_{00} & \pi_{01} \\
\pi_{10} & \pi_{11}
\end{array}\right] .
$$

At time $n$, the channel can be characterized by the following equation:

$$
Z_{n}=Y_{n} \oplus E_{n}
$$

where $\oplus$ denotes binary addition, $E_{n}$ denotes the independent and identically distributed (i.i.d.) binary noise with $p_{E}(0)=$ $1-\varepsilon$ and $p_{E}(1)=\varepsilon$, and $Z_{n}$ denotes the corrupted output. Then $\left(Y_{n}, E_{n}\right)$ is jointly Markov, so $\left\{Z_{n}\right\}$ is a hidden Markov chain with the corresponding

$$
\Delta=\left[\begin{array}{llll}
\pi_{00}(1-\varepsilon) & \pi_{00} \varepsilon & \pi_{01}(1-\varepsilon) & \pi_{01} \varepsilon \\
\pi_{00}(1-\varepsilon) & \pi_{00} \varepsilon & \pi_{01}(1-\varepsilon) & \pi_{01} \varepsilon \\
\pi_{10}(1-\varepsilon) & \pi_{10} \varepsilon & \pi_{11}(1-\varepsilon) & \pi_{11} \varepsilon \\
\pi_{10}(1-\varepsilon) & \pi_{10} \varepsilon & \pi_{11}(1-\varepsilon) & \pi_{11} \varepsilon
\end{array}\right] ;
$$

here, $\Phi$ maps states 1 and 4 to 0 and maps states 2 and 3 to 1 . This class of hidden Markov chains has been studied extensively (e.g., [12], [20]).

By Theorem 1.1, when $\varepsilon$ and $\pi_{i j}$ 's are positive, the entropy rate $H(Z)$ is analytic as a function of $\varepsilon$ and $\pi_{i j}$ 's. This still holds when $\varepsilon=0$ and the $\pi_{i j}$ 's are positive, because in this case, we have

$$
\Delta=\left[\begin{array}{llll}
\pi_{00} & 0 & \pi_{01} & 0 \\
\pi_{00} & 0 & \pi_{01} & 0 \\
\pi_{10} & 0 & \pi_{11} & 0 \\
\pi_{10} & 0 & \pi_{11} & 0
\end{array}\right]
$$

\section{DOMAIN OF ANALYTICITY}

Suppose $\Delta$ is analytically parameterized by a vector variable $\vec{\varepsilon}$, and Conditions 1 and 2 in Theorem 1.1 are satisfied at $\vec{\varepsilon}=\vec{\varepsilon}_{0}$. In principle, the proof of Theorem 1.1 determines a neighborhood $\Omega_{\mathbb{C}}(r)$ of $\vec{\varepsilon}_{0}$ on which the entropy rate is analytic. Specifically, if one can find $\rho, r$ and $R$ such that all of the following hold, then the entropy rate is analytic on $\Omega_{\mathbb{C}}(r)$.

1. Find $\rho$ such that each $f_{a}^{\varepsilon_{0}}$ is a Euclidean $\rho$-contraction on each $W_{b}$. Then choose positive $r, R$ such that for all $\vec{\varepsilon} \in \Omega_{\mathbb{C}}(r)$, each $f_{a}^{\overrightarrow{\vec{\varepsilon}}}$ is a Euclidean $\rho$-contraction on each $N_{b}(R)$ (see (4.8)).

2. Next find $r$ smaller (if necessary) such that for all $\vec{\varepsilon} \in$ $\Omega_{\mathbb{C}}(r)$, the image of the stationary vector of $\Delta^{\vec{\varepsilon}}$, under any composition of the mappings $\left\{f_{a}^{\varepsilon}\right\}$, stays within $\cup_{b} N_{b}(R)$ (see (4.9)). Note that the argument in the proof shows that this holds if (4.10) and (4.11) hold.

3 . Finally, find $r, R$ such that the sum of the absolute values of the complexified conditional probabilities, conditioned on any given past symbol sequence, is $<1 / \rho$, and similarly for the sum of the absolute values of the complexified stationary probabilities (see (4.17) and (4.18)).

In fact, the proof shows that one can always find such $\rho, r, R$, but in Condition 1 above one may need to replace $f_{a}$ 's by all $n$-fold compositions of the $f_{a}$ 's, for some $n$.

Recall from Example 4.1 the family of hidden Markov chains $Z^{\varepsilon}$ determined by passing a binary Markov chain through a 
binary-symmetric channel with crossover probability $\varepsilon$. Recall that $H\left(Z^{\varepsilon}\right)$ is an analytic function of $\varepsilon$ at $\varepsilon=0$ when the Markov transition probabilities are all positive. We shall determine a complex neighborhood of 0 such that the entropy rate, as a function of $\varepsilon$, is analytic on this neighborhood.

Let $u_{n}=p\left(y_{n}=0 \mid z_{1}^{n}\right)$ and $v_{n}=p\left(y_{n}=1 \mid z_{1}^{n}\right)$. For $z_{n+1}=1$ we have

$$
\begin{aligned}
u_{n+1} & =\frac{\varepsilon\left(\pi_{00} u_{n}+\pi_{10} v_{n}\right)}{\varepsilon\left(\pi_{00} u_{n}+\pi_{10} v_{n}\right)+(1-\varepsilon)\left(\pi_{01} u_{n}+\pi_{11} v_{n}\right)} \\
v_{n+1} & =\frac{(1-\varepsilon)\left(\pi_{01} u_{n}+\pi_{11} v_{n}\right)}{\varepsilon\left(\pi_{00} u_{n}+\pi_{10} v_{n}\right)+(1-\varepsilon)\left(\pi_{01} u_{n}+\pi_{11} v_{n}\right)} .
\end{aligned}
$$

Since $u_{n}+v_{n}=1, u_{n+1}$ is a function of $u_{n}$; let $g_{1}$ denote this function.

For $z_{n+1}=0$ we have

$$
\begin{aligned}
u_{n+1} & =\frac{(1-\varepsilon)\left(\pi_{00} u_{n}+\pi_{10} v_{n}\right)}{(1-\varepsilon)\left(\pi_{00} u_{n}+\pi_{10} v_{n}\right)+\varepsilon\left(\pi_{01} u_{n}+\pi_{11} v_{n}\right)} \\
v_{n+1} & =\frac{\varepsilon\left(\pi_{01} u_{n}+\pi_{11} v_{n}\right)}{(1-\varepsilon)\left(\pi_{00} u_{n}+\pi_{10} v_{n}\right)+\varepsilon\left(\pi_{01} u_{n}+\pi_{11} v_{n}\right)} .
\end{aligned}
$$

Again, $u_{n+1}$ is a function of $u_{n}$; let $g_{0}$ denote this function.

And for the conditional probability, we have

$$
\begin{aligned}
p\left(z_{n}=0 \mid z_{1}^{n-1}\right)=\left((1-\varepsilon) \pi_{00}+\right. & \left.\varepsilon \pi_{01}\right) u_{n} \\
& +\left((1-\varepsilon) \pi_{10}+\varepsilon \pi_{11}\right) v_{n} .
\end{aligned}
$$

Since $u_{n}+v_{n}=1, p\left(z_{n}=0 \mid z_{1}^{n-1}\right)$ is a function of $u_{n}$; let $r_{0}$ denote this function. And

$$
\begin{aligned}
p\left(z_{n}=1 \mid z_{1}^{n-1}\right)=\left(\varepsilon \pi_{00}+(1-\varepsilon)\right. & \left.\pi_{01}\right) u_{n} \\
& +\left(\varepsilon \pi_{10}+(1-\varepsilon) \pi_{11}\right) v_{n} .
\end{aligned}
$$

Again, $p\left(z_{n}=1 \mid z_{1}^{n-1}\right)$ is a function of $u_{n}$; let $r_{1}$ denote this function.

Note that $g_{0}, g_{1}, r_{0}, r_{1}$ are all implicitly parameterized by $\varepsilon$. The stationary vector $\left(\pi_{0}, \pi_{1}\right)$ of $Y$, which does not depend on $\varepsilon$, is equal to $\left(\pi_{10} /\left(\pi_{10}+\pi_{01}\right), \pi_{01} /\left(\pi_{10}+\pi_{01}\right)\right)$.

We shall choose $\rho$ with $0<\rho<1, r>0$ and $R>0$ such that for all $\varepsilon$ with $|\varepsilon|<r$
1. $g_{0}$ and $g_{1}$ are $\rho$-contraction mappings on $R$-neighborhoods of 0 and 1 in the complex plane;

2. the set of all $\left.\left\{g_{a_{n}} \circ g_{a_{n-1}} \circ \cdots \circ g_{a_{1}}\left(\pi_{0}\right)\right\}\right)$ are within the $R$-neighborhoods of 0 and 1 ;

3. $\left|r_{0}(u)\right|+\left|r_{1}(u)\right|<1 / \rho$ for $u$ in $R$-neighborhoods of 0 and 1 in the complex plane.

By the general principle above, the entropy rate should be analytic on $|\varepsilon|<r$.

More concretely, Conditions 1, 2, and 3 translate to (here $\rho<1)$ :

1. $\left|g_{0}^{\prime}(u)\right|<\rho,\left|g_{1}^{\prime}(u)\right|<\rho$ on $(|\varepsilon|<r$ and $|u|<R)$ and $(|\varepsilon|<r$ and $|1-u|<R)$;

2. $\max \left\{\left|g_{0}(0)-1\right|,\left|g_{0}(1)-1\right|,\left|g_{1}(0)\right|,\left|g_{1}(1)\right|\right\}<R(1-\rho)$ on $|\varepsilon|<r$ (this follows from (4.10); (4.11) is trivial since the stationary vector of $Y$ does not depend on $\varepsilon$ );

3. $\left|r_{0}(u)\right|+\left|r_{1}(u)\right|<1 / \rho$ on $(|\varepsilon|<r$ and $|u|<R)$ and $(|\varepsilon|<r$ and $|1-u|<R)$

A straightforward computation shows that the conditions shown at the bottom of the page guarantee Conditions 1, 2, 3 .

In other words, for given $\rho$ with $0<\rho<1$, choose $r$ and $R$ to satisfy all the constraints above. Then the entropy rate is an analytic function of $\varepsilon$ on $|\varepsilon|<r$.

Let $\pi_{00}=\pi_{11}=p$ and $\pi_{01}=\pi_{10}=1-p$. We plot lower bounds on radius of convergence of $H(Z)$ (as a function of $\varepsilon$ ) against $p$ in Fig. 1. For a fixed $p$, the lower bound is obtained by randomly generating many 3 -tuples $(r, R, \rho)$ and taking the maximal $r$ from the 3 -tuples which satisfy the inequality conditions above. One can see in the plot that as $p$ goes to 0.5 , the lower bound is rapidly increasing. This is not surprising, since when $p=0.5$, the corresponding entropy rate is a constant function of $\varepsilon$, and thus the radius of convergence is $\infty$.

\section{RELAXED CONDITIONS}

We do not know a complete set of necessary and sufficient conditions on $\Delta$ and $\Phi$ that guarantee analyticity of entropy rate. However, in this section, we show how the hypotheses in Theorem 1.1 can be relaxed and still guarantee analyticity. We then give several examples. In Section VII, we do give a complete set

$$
\begin{aligned}
0 & \leq \frac{\sqrt{r(r+1)\left|-\pi_{00} \pi_{11}+\pi_{10} \pi_{01}\right|}}{\pi_{11}-\left|\pi_{10}-\pi_{11}\right| r-\left(\left|\pi_{00}-\pi_{10}-\pi_{01}+\pi_{11}\right| r+\left|\pi_{01}-\pi_{11}\right|\right) R}<\sqrt{\rho} \\
0 & \leq \frac{\sqrt{r(r+1)\left|-\pi_{00} \pi_{11}+\pi_{10} \pi_{01}\right|}}{\pi_{01}-\left|\pi_{00}-\pi_{01}\right| r-\left(\left|\pi_{00}-\pi_{10}-\pi_{01}+\pi_{11}\right| r+\left|\pi_{01}-\pi_{11}\right|\right) R}<\sqrt{\rho} \\
0 \leq & \sqrt{r(r+1)\left|-\pi_{11} \pi_{00}+\pi_{01} \pi_{10}\right|} \\
0 \leq & \frac{\sqrt{r(r+1)\left|-\pi_{11} \pi_{00}+\pi_{01} \pi_{10}\right|}}{\pi_{00}-\left|\pi_{01}-\pi_{00}\right| r-\left(\left|\pi_{00}-\pi_{10}+\pi_{11}-\pi_{01}\right| r+\left|\pi_{10}-\pi_{00}\right|\right) R}<\sqrt{\rho} \\
0 \leq & \frac{r \pi_{10}-\pi_{10} \mid r-\left(\left|\pi_{00}-\pi_{10}+\pi_{11}-\pi_{01}\right| r+\left|\pi_{10}-\pi_{00}\right|\right) R}{\pi_{01}-\left|\pi_{00}-\pi_{01}\right| r}<\sqrt{\rho} \\
0 \leq & \frac{r \pi_{11}}{\pi_{10}-\left|\pi_{11}-\pi_{10}\right| r}<R(1-\rho), 0 \leq \frac{r \pi_{01}}{\pi_{00}-\left|\pi_{01}-\pi_{00}\right| r}<R(1-\rho) \\
& 2\left(\left|\pi_{00}-\pi_{01}-\pi_{10}+\pi_{11}\right| r+\left|\pi_{01}-\pi_{11}\right|\right) R+2\left|\pi_{10}-\pi_{11}\right| r+1<1 / \rho \\
& 2\left(\left|\pi_{10}-\pi_{11}-\pi_{00}+\pi_{01}\right| r+\left|\pi_{11}-\pi_{01}\right|\right) R+2\left|\pi_{00}-\pi_{01}\right| r+1<1 / \rho .
\end{aligned}
$$




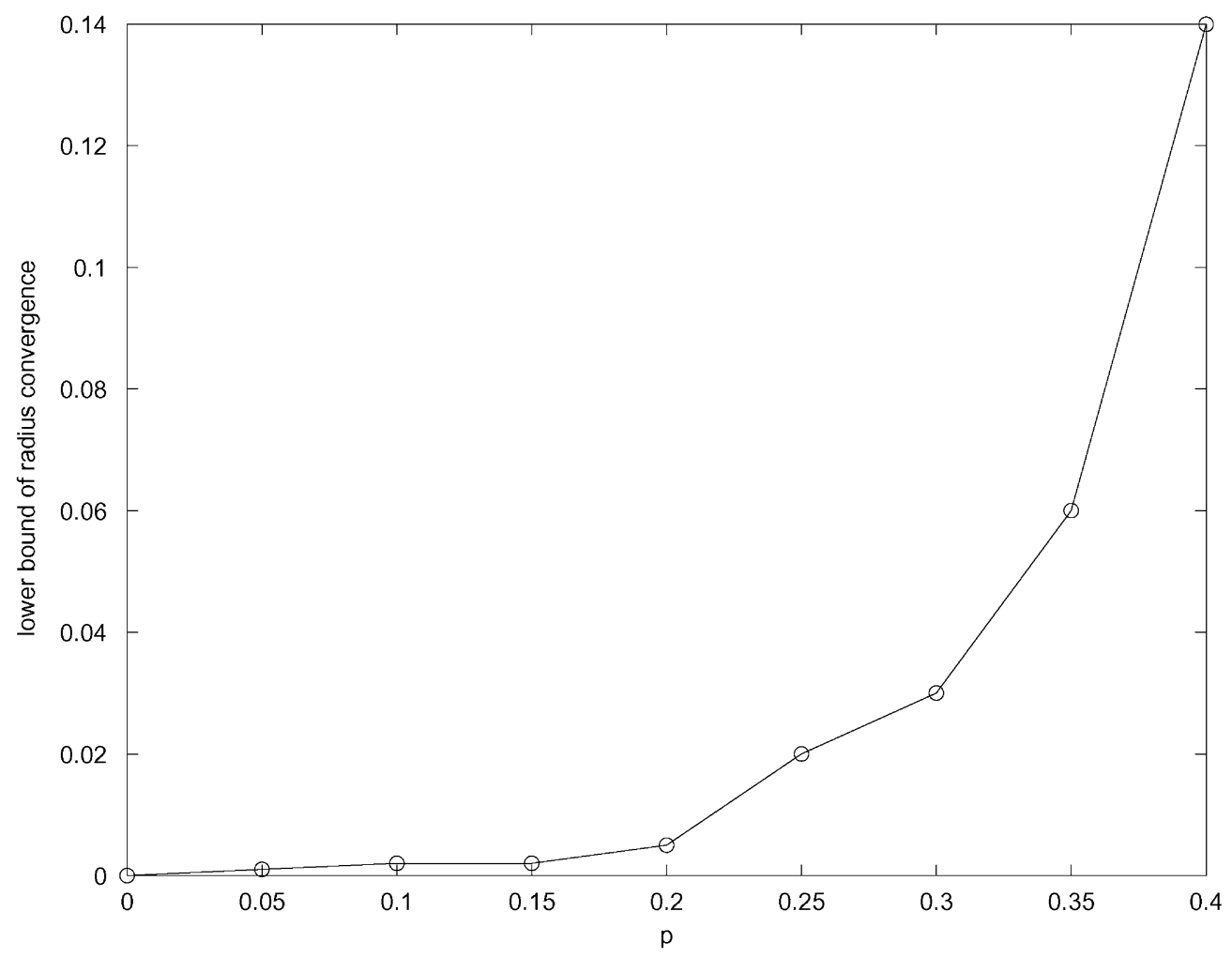

Fig. 1. Lower bound on radius of convergence as a function of $p$.

of necessary and sufficient conditions for a very special class of hidden Markov chains.

In this section, we assume that $\Delta$ has a simple maximum eigenvalue 1 ; this implies that $\Delta$ has a unique stationary vector $\vec{s}$.

For a mapping $f$ from $W_{b}$ to $W$ and $w \in W_{b}$. Let $f^{\prime}$ denote the first derivative of $f$ at $w$ restricted to the subspace spanned by directions parallel to the simplex $W_{b}$ and let $\|\cdot\|$ denote the Euclidean norm of a linear mapping. We say that $\left\{f_{a}: a \in\{1,2, \ldots, A\}\right\}$ is eventually contracting at $w \in W_{b}$ if there exists $n$ such that for any $a_{0}, a_{1}, \ldots, a_{n} \in\{1,2, \ldots, A\}$, $\left\|\left(f_{a_{n}} \circ f_{a_{n-1}} \circ \cdots \circ f_{a_{0}}\right)^{\prime}(w)\right\|$ is strictly less than 1 . We say that $\left\{f_{a}: a \in\{1,2, \ldots, A\}\right\}$ is contracting at $w \in W_{b}$ if it is eventually contracting at $w$ with $n=0$. Using the mean value theorem, one can show that if $\left\{f_{a}: a \in\{1,2, \ldots, A\}\right\}$ is contracting at each $w$ in a compact convex subset $K$ of $W_{b}$ then each $f_{a}$ is a contraction mapping on $K$.

Let $L$ denote the limit set of

$$
\begin{aligned}
& \left\{\left(f_{a_{n}} \circ f_{a_{n-1}} \circ \cdots \circ f_{a_{0}}\right)(\vec{s}):\right. \\
& \left.a_{1}, a_{2}, \ldots, a_{n} \in\{1,2, \ldots, A\}, n \geq 0\right\} .
\end{aligned}
$$

Theorem 6.1: If at $\Delta=\hat{\Delta}$

1. 1 is a simple eigenvalue for $\hat{\Delta}$;

2. for every $a$ and all $w$ in $L, r_{a}(w)>0$;

3. for every $b,\left\{f_{a}: a \in\{1,2, \ldots, A\}\right\}$ is eventually contracting at all $w$ in the convex hull of the intersection of $L$ and $W_{b}$;

then $H(Z)$ is analytic at $\Delta=\hat{\Delta}$.

Proof: Let $\mathcal{X}$ denote the right infinite shift space $\left\{a_{0}^{\infty}: a_{i} \in\{1,2, \ldots, A\}\right\}$. Let $L_{b}^{\delta}$ be the set of all points in
$W_{b}$ of distance at most $\delta$ from $L \cap W_{b}$, and let $L^{\delta}=\cup_{b} L_{b}^{\delta}$. Choose $\delta$ so small that

- for every $a \in\{1,2, \ldots, A\}$ and $w$ in $L^{\delta}, r_{a}(w)>0$ and

- for every $b,\left\{f_{a}: a \in\{1,2, \ldots, A\}\right\}$ is eventually contracting at all $w$ in the convex hull $K_{b}^{\delta}$ of $L_{b}^{\delta}$.

Since $K_{b}^{\delta}$ is compact, there exists $n$ such that for any $a_{0}, a_{1}, \ldots, a_{n} \in\{1,2, \ldots, A\}$ and any $w \in K_{b}^{\delta}$, $\left\|\left(f_{a_{n}} \circ f_{a_{n-1}} \circ \cdots \circ f_{a_{0}}\right)^{\prime}(w)\right\|$ is strictly less than 1 . For simplicity, we may assume that $\left\{f_{a}\right\}$ is contracting on $K_{b}^{\delta}$, and so each $f_{a}$ is a contraction mapping on $K_{b}^{\delta}$. Since $L_{b}^{\delta} \subseteq K_{b}^{\delta}$, it follows that $f_{a}\left(L^{\delta}\right) \subseteq L^{\delta}$.

For any $c_{0}^{\infty} \in \mathcal{X}$, there exists $n$ such that

$$
\left\{\left(f_{c_{n}} \circ f_{c_{n-1}} \circ \cdots \circ f_{c_{0}}\right)(\vec{s})\right\} \in L^{\delta} .
$$

Let $\mathcal{X}_{c_{0}^{\infty}}^{n}$ denote the cylinder set

$$
\left\{a_{0}^{\infty}: a_{0}=c_{0}, a_{1}=c_{1}, \ldots, a_{n}=c_{n}\right\} .
$$

Since $f_{a}\left(L^{\delta}\right) \subset L^{\delta}$, we conclude that for any $a_{0}^{\infty} \in \mathcal{X}_{c_{0}^{\infty}}^{n}$ and all

$$
m \geq n,\left\{\left(f_{a_{m}} \circ f_{a_{m-1}} \circ \cdots \circ f_{a_{0}}\right)(\vec{s})\right\} \in L^{\delta} .
$$

By the compactness of $\mathcal{X}$, we can find finitely many such cylinder sets to cover $\mathcal{X}$. Consequently, we can find $n$ such that for any $a_{0}^{\infty} \in \mathcal{X}$ and any $m \geq n$, we have $\left\{\left(f_{a_{m}} \circ f_{a_{m-1}} \circ \cdots \circ f_{a_{0}}\right)(\vec{s})\right\} \in L^{\delta}$. We can now apply the proof of Theorem 1.1-namely, we can use the contraction (along any symbolic sequence $\left.z_{-n}^{0}\right)$ to extend $H_{n}(Z)=H\left(Z_{0} \mid Z_{-n}^{-1}\right)$ from real to complex and prove the uniform convergence of $H_{n}(Z)$ to $H(Z)$ in complex parameter space. 


\section{Remark 6.2:}

1) If $\hat{\Delta}$ has a strictly positive column (or more generally, there is a $j$ such that for all $i$, there exists $n$ such that $\hat{\Delta}_{i j}^{n}>0$ ), then Condition 1 of Theorem 6.1 holds by Perron-Frobenius theory.

2) If for each symbol $a, \hat{\Delta}_{a}$ is row allowable (i.e., no row is all zero), then $r_{a}(w)>0$ for all $w \in W$ and so Condition 2 of Theorem 6.1 holds.

Theorem 6.1 relaxes the positivity assumptions of Theorem 1.1. Indeed, given Conditions 1 and 2 of Theorem 1.1, by Remark 6.2, Conditions 1 and 2 of Theorem 6.1 hold. For Condition 3 of Theorem 6.1, first observe that $L$ is contained in $\cup_{b} f_{b}(W)$. Using the equivalence of the Euclidean metric and the Hilbert metric, Proposition 2.2 shows that for every $b$, $\left\{f_{a}: a \in\{1,2, \ldots, A\}\right\}$ is eventually contracting on $f_{b}(W)$, which is a convex set containing the intersection of $L$ and $W_{b}$.

Theorem 6.1 also applies to many cases not covered by Theorem 1.1. Suppose that some column of $\hat{\Delta}$ is strictly positive and each $\hat{\Delta}_{a}$ is row allowable. By Remark 6.2, Theorem 6.1 applies whenever we can guarantee Condition 3. For this, it is sufficient to check that for each $a, b, f_{a}$ is a contraction, with respect to the Euclidean metric, on the convex hull of the intersection of $L$ with each $W_{b}$. This can be done by explicitly computing derivatives. This is illustrated by the following example.

Example 6.3: Consider a hidden Markov chain $Z$ defined by

$$
\hat{\Delta}=\left[\begin{array}{cccc}
a_{11} & a_{12} & a_{13} & a_{14} \\
a_{21} & a_{22} & a_{23} & a_{24} \\
a_{31} & a_{32} & a_{33} & a_{34} \\
a_{41} & a_{42} & a_{43} & a_{44}
\end{array}\right]
$$

with $\Phi(1)=\Phi(2)=0$ and $\Phi(3)=\Phi(4)=1$. We assume that some column of $\hat{\Delta}$ is strictly positive and both $\hat{\Delta}_{0}$ and $\hat{\Delta}_{1}$ are row allowable.

Parameterize $W_{0}$ by $(y, 1-y, 0,0)$ and parameterize $W_{1}$ by $(0,0, y, 1-y)$ (with $y \in[0,1])$. We can explicitly compute the derivatives of $f_{0}$ and $f_{1}$ with respect to $y$

$$
\begin{aligned}
\left.f_{0}^{\prime}\right|_{(y, 1-y, 0,0)} & =\frac{a_{11} a_{22}-a_{12} a_{21}}{\left(\left(a_{11}+a_{12}-a_{21}-a_{22}\right) y+a_{21}+a_{22}\right)^{2}} \\
\left.f_{0}^{\prime}\right|_{(0,0, y, 1-y)} & =\frac{a_{31} a_{42}-a_{32} a_{41}}{\left(\left(a_{31}+a_{32}-a_{41}-a_{42}\right) y+a_{41}+a_{42}\right)^{2}} \\
\left.f_{1}^{\prime}\right|_{(y, 1-y, 0,0)} & =\frac{a_{13} a_{24}-a_{14} a_{23}}{\left(\left(a_{13}+a_{14}-a_{23}-a_{24}\right) y+a_{23}+a_{24}\right)^{2}} \\
\left.f_{1}^{\prime}\right|_{(0,0, y, 1-y)} & =\frac{a_{33} a_{44}-a_{34} a_{43}}{\left(\left(a_{33}+a_{34}-a_{43}-a_{44}\right) y+a_{43}+a_{44}\right)^{2}} .
\end{aligned}
$$

Note that the row allowability condition guarantees that the denominators in these expressions never vanish.

Choose $a_{i j}$ 's such that each of these derivatives is less than 1 ; then we conclude that the entropy rate is analytic at $\hat{\Delta}$. One way to do this is to make each of the $2 \times 2$ upper/lower left/right matrices singular.

Or choose the $a_{i j}$ 's such that

$$
\hat{\Delta}=\left[\begin{array}{cccc}
\alpha_{1} & * & \beta_{1} & 0 \\
0 & \alpha_{2} & 0 & \beta_{2} \\
\lambda_{1} & * & \eta_{1} & 0 \\
0 & \lambda_{2} & 0 & \eta_{2}
\end{array}\right]
$$

where $0<\alpha_{1}<\alpha_{2}, 0<\beta_{1}<\beta_{2}, 0<\lambda_{1}<\lambda_{2}, 0<\eta_{1}<$ $\eta_{2}$ and $*$ denote a real positive number (note that Theorem 1.1 does not apply for this special case). Let $\left(s_{2}, s_{4}\right)$ be the Perron eigenvector of the stochastic matrix

$$
\left[\begin{array}{ll}
\alpha_{2} & \beta_{2} \\
\lambda_{2} & \eta_{2}
\end{array}\right]
$$

Then $\vec{s}=\left(0, s_{2}, 0, s_{4}\right)$ is the stationary vector of $\Delta$ corresponding to the simple eigenvalue 1 . Let $w_{0}=(0,1,0,0)$ and $w_{1}=(0,0,0,1)$. One checks that for $n \geq 0, f_{a_{n}} \circ f_{a_{n-1}} \circ \cdots \circ$ $f_{a_{0}}(\vec{s})=w_{a_{n}}$. Therefore, $L$ consists of $\left\{w_{0}, w_{1}\right\}$. Using the expressions above, we see that

$$
\begin{aligned}
& \left.f_{0}^{\prime}\right|_{w_{0}}=\alpha_{1} / \alpha_{2}<1 \\
& \left.f_{0}^{\prime}\right|_{w_{1}}=\lambda_{1} / \lambda_{2}<1 \\
& \left.f_{1}^{\prime}\right|_{w_{0}}=\beta_{1} / \beta_{2}<1 \\
& \left.f_{1}^{\prime}\right|_{w_{1}}=\eta_{1} / \eta_{2}<1 .
\end{aligned}
$$

So, $f_{0}$ and $f_{1}$ are contraction mappings at $\left\{w_{0}, w_{1}\right\}$, and so Condition 3 holds. Thus, the entropy rate $H(Z)$ is analytic at $\hat{\Delta}$.

\section{Hidden Markov Chains With Unambiguous Symbol}

Definition 7.1: A symbol $a$ is called unambiguous if $\Phi^{-1}(a)$ contains only one element.

Remark 7.2: Note that unambiguous symbol is referred to as "singleton clump" in some ergodic theory work, such as [24].

When an unambiguous symbol is present, the entropy rate can be expressed in a simple way: letting $a_{1}$ be an unambiguous symbol

$$
\begin{aligned}
H(Z)=\sum_{a_{i_{j}} \neq a_{1}} p\left(a_{i_{n}} a_{i_{n-1}}\right. & \left.\cdots a_{i_{2}} a_{1}\right) \\
\times & \\
\times & H\left(z \mid a_{i_{n}} a_{i_{n-1}} \cdots a_{i_{2}} a_{1}\right) .
\end{aligned}
$$

In this section, we focus on the case of a binary hidden Markov chain, in which 0 is unambiguous. Then, we can rewrite (7.21) as

$$
\begin{aligned}
H\left(Z^{\vec{\varepsilon}}\right)=p^{\vec{\varepsilon}}(0) H^{\vec{\varepsilon}}(z \mid 0)+p^{\vec{\varepsilon}}(10) H^{\vec{\varepsilon}}(z \mid 10)+\cdots \\
+p^{\vec{\varepsilon}}\left(1^{(n)} 0\right) H^{\vec{\varepsilon}}\left(z \mid 1^{(n)} 0\right)+\cdots
\end{aligned}
$$

where $1^{(n)}$ denotes the sequence of $n 1$ 's and

$$
\begin{aligned}
H^{\vec{\varepsilon}}\left(z \mid 1^{(n)} 0\right)=-p^{\vec{\varepsilon}}\left(0 \mid 1^{(n)} 0\right) & \log p^{\vec{\varepsilon}}\left(0 \mid 1^{(n)} 0\right) \\
& -p^{\vec{\varepsilon}}\left(1 \mid 1^{(n)} 0\right) \log p^{\vec{\varepsilon}}\left(1 \mid 1^{(n)} 0\right) .
\end{aligned}
$$

Example 7.3: Fix $a, b, \ldots, h>0$ and for $\varepsilon \geq 0$ let

$$
\Delta(\varepsilon)=\left[\begin{array}{ccc}
\varepsilon & a-\varepsilon & b \\
g & c & d \\
h & e & f
\end{array}\right] .
$$

Assume $a, b, \ldots, h>0$ are chosen such that $\Delta(\varepsilon)$ is stochastic. The symbols of the Markov chain are the matrix indices $\{1,2,3\}$. Let $Z^{\varepsilon}$ be the binary hidden Markov chain 
defined by $\Phi(1)=0$ and $\Phi(2)=\Phi(3)=1$. We claim that $H\left(Z^{\varepsilon}\right)$ is not analytic at $\varepsilon=0$.

Let $\pi(\varepsilon)$ be the stationary vector of $\Delta(\varepsilon)$ (which is unique since $\Delta(\varepsilon)$ is irreducible). Observe that

$$
p^{\varepsilon}(0)=\pi_{1}(\varepsilon), p^{\varepsilon}(00)=\pi_{1}(\varepsilon) \varepsilon
$$

and for $n \geq 1$

$$
p^{\varepsilon}\left(1^{(n)} 0\right)=\pi_{1}(\varepsilon)(a-\varepsilon, b)\left[\begin{array}{ll}
c & d \\
e & f
\end{array}\right]^{n-1}\left(\begin{array}{l}
1 \\
1
\end{array}\right) .
$$

Since $\Delta(\varepsilon)$ is irreducible, $\pi(\varepsilon)$ is analytic in $\varepsilon$ and positive. Now

$$
p^{\varepsilon}(0) H^{\varepsilon}(z \mid 0)=-p^{\varepsilon}(00) \log p^{\varepsilon}(0 \mid 0)-p^{\varepsilon}(10) \log p^{\varepsilon}(1 \mid 0) .
$$

The first term in (7.23) is

$$
-p^{\varepsilon}(00) \log p^{\varepsilon}(0 \mid 0)=-\pi_{1}(\varepsilon) \varepsilon \log \varepsilon
$$

which is not analytic (or even differentiable at $\varepsilon=0$ ). The second term in (7.23) is

$-p^{\varepsilon}(10) \log p^{\varepsilon}(1 \mid 0)=-\pi_{1}(\varepsilon)(a-\varepsilon+b) \log \left(\pi_{1}(\varepsilon)(a-\varepsilon+b)\right)$

which is analytic at $\varepsilon=0$. Thus, $H^{\varepsilon}(z \mid 0)$ is not analytic at $\varepsilon=0$. Similarly, it can be shown that all of the terms of (7.22), other than $H^{\varepsilon}(z \mid 0)$, are analytic at $\varepsilon=0$. Since the matrix

$$
\left[\begin{array}{ll}
c & d \\
e & f
\end{array}\right]
$$

has spectral radius $<1$, the terms of (7.22) decay exponentially; it follows that the infinite sum of these terms is analytic. Thus, $H\left(Z^{\varepsilon}\right)$ is the sum of two functions of $\varepsilon$, one of which is analytic and the other is not analytic at $\varepsilon=0$. Thus, $H\left(Z^{\varepsilon}\right)$ is not analytic at $\varepsilon=0$.

Example 7.4: Fix $a, b, \ldots, g>0$ and consider the stochastic matrix

$$
\Delta(\varepsilon)=\left[\begin{array}{ccc}
e & a & b \\
f-\varepsilon & c & \varepsilon \\
g & 0 & d
\end{array}\right] .
$$

The symbols of the Markov chain are the matrix indices $\{1,2,3\}$. Again let $Z^{\varepsilon}$ be the binary hidden Markov chain defined by $\Phi(1)=0$ and $\Phi(2)=\Phi(3)=1$. We show that $H\left(Z^{\varepsilon}\right)$ is analytic at $\varepsilon=0$ when $c \neq d$, and not analytic when $c=d$. Note that

$$
p^{\varepsilon}(0)=\pi_{1}(\varepsilon)
$$

and for $n \geq 1$

$$
p^{\varepsilon}\left(1^{(n)} 0\right)=\pi_{1}(\varepsilon)(a, b)\left[\begin{array}{ll}
c & \varepsilon \\
0 & d
\end{array}\right]^{n-1}\left(\begin{array}{l}
1 \\
1
\end{array}\right) .
$$

When $c \neq d$, we assume $c>d$, then

$$
\left[\begin{array}{ll}
c & \varepsilon \\
0 & d
\end{array}\right]^{n}=\left[\begin{array}{cc}
c^{n} & \varepsilon c^{n-1} \frac{1-(d / c)^{n}}{1-d / c} \\
0 & d^{n}
\end{array}\right] .
$$

Since $\Delta(\varepsilon)$ is irreducible, $\pi(\varepsilon)$ is analytic in $\varepsilon$ and positive. Simple computation leads to

$$
\begin{aligned}
& p^{\varepsilon}\left(1 \mid 1^{(n)} 0\right) \\
&=\left(a c^{n}+a \varepsilon c^{n-1} \frac{1-(d / c)^{n}}{1-d / c}+b d^{n}\right) / \\
&\left(a c^{n-1}+a \varepsilon c^{n-2} \frac{1-(d / c)^{n-1}}{1-d / c}+b d^{n-1}\right) \\
&=\left(a c^{2}+a \varepsilon c \frac{1-(d / c)^{n}}{1-d / c}+b d^{2}(d / c)^{n-2}\right) / \\
&\left(a c+\varepsilon \frac{1-(d / c)^{n-1}}{1-d / c}+b d(d / c)^{n-2}\right)
\end{aligned}
$$

and

$$
\begin{aligned}
& p^{\varepsilon}\left(0 \mid 1^{(n)} 0\right) \\
&=\left((f-\varepsilon) a c^{n-1}\right. \\
&\left.+g\left(a \varepsilon c^{n-2} \frac{1-(d / c)^{n-1}}{1-d / c}+b d^{n-1}\right)\right) / \\
&\left(a c^{n-1}+a \varepsilon c^{n-2} \frac{1-(d / c)^{n-1}}{1-d / c}+b d^{n-1}\right) \\
&=\left((f-\varepsilon) a c+g\left(a \varepsilon \frac{1-(d / c)^{n-1}}{1-d / c}+b d(d / c)^{n-2}\right)\right) / \\
&\left(a c+\varepsilon \frac{1-(d / c)^{n-1}}{1-d / c}+b d(d / c)^{n-2}\right) .
\end{aligned}
$$

In this case, all terms are analytic. Again since

$$
\left[\begin{array}{ll}
c & \varepsilon \\
0 & d
\end{array}\right]
$$

has spectral radius $<1$, the term $p^{\varepsilon}\left(1^{(n)} 0\right) H^{\varepsilon}\left(z \mid 1^{(n)} 0\right)$ is exponentially decaying with respect to $n$. Therefore, the infinite sum of these terms is also analytic, and so the entropy rate is a real analytic function of $\varepsilon$.

When $c=d$, we have

$$
\begin{aligned}
p^{\varepsilon}\left(1 \mid 1^{(n)} 0\right)= & \left(a c^{n+1}+a \varepsilon(n+1) c^{n}+b c^{n+1}\right) / \\
& \times\left(a c^{n}+a \varepsilon n c^{n-1}+b c^{n}\right) \\
= & \left(a c^{2}+a \varepsilon(n+1) c+b c^{2}\right) /(a c+a \varepsilon n+b c),
\end{aligned}
$$

and

$$
\begin{aligned}
p^{\varepsilon}\left(0 \mid 1^{(n)} 0\right)= & \left((f-\varepsilon) a c^{n}+g a \varepsilon n c^{n-1}+g b c^{n}\right) / \\
& \times\left(a c^{n}+a \varepsilon n c^{n-1}+b c^{n}\right) \\
= & ((f-\varepsilon) a c+g a \varepsilon n+g b c) /(a c+a \varepsilon n+b c) .
\end{aligned}
$$

For any $n$, consider a small neighborhood $N_{n}$ of $-(a+b) c / a n$ in $\mathbb{C}$ such that $-(a+b) c / a j \in N_{n}$ only holds for $j=n$. When $\varepsilon \rightarrow-(a+b) c / a n$, the complexified term $p^{\varepsilon}\left(1^{(n)} 0\right) H^{\varepsilon}\left(z \mid 1^{(n)} 0\right) \rightarrow \infty$. Meanwhile, the sum of all the other terms can be analytically extended to $N_{n}$ (from any path $I$ from a positive $\varepsilon$ to $-(a+b) c / a n$ with $-(a+b) c / a j \notin I$ for $j \neq n$ ). Thus, by the uniqueness of analytic continuation of $H\left(Z^{\varepsilon}\right)$, we conclude that $H\left(Z^{\varepsilon}\right)$ blows up when one approaches $-(a+b) c / a n$ and therefore is not analytic at $\varepsilon=0$ (although it is smooth from the right at $\varepsilon=0$ ). 
The two examples above show that under certain conditions the entropy rate of a binary hidden Markov chain with unambiguous symbol can fail to be analytic at the boundary. We now show that these examples typify all the types of failures of analyticity at the boundary (in the case of a binary hidden Markov chains with an unambiguous symbol).

We will need the following result.

Lemma 7.5: Let $A(\vec{\varepsilon})$ be an analytic parameterization of complex matrices. Let $\lambda$ be the spectral radius of $A\left(\vec{\varepsilon}_{0}\right)$. Then for any $\eta>0$, there exists a complex neighborhood $\Omega$ of $\vec{\varepsilon}_{0}$ and positive constant $C$ such that for all $\vec{\varepsilon} \in \Omega$ and all $i, j, k$

$$
\left|A_{i j}^{k}(\vec{\varepsilon})\right| \leq C(\lambda+\eta)^{k}
$$

Proof: Following [29], we consider

$$
(I-z A)^{-1}=I+z A+z^{2} A^{2}+\cdots .
$$

And

$$
\begin{aligned}
(I-z A)^{-1} & =\frac{\operatorname{Adj}(I-z A)}{\operatorname{det}(I-z A)} \\
& =\frac{\operatorname{Adj}(I-z A)}{\left(1-\lambda_{1} z\right)\left(1-\lambda_{2} z\right) \cdots\left(1-\lambda_{n} z\right)}
\end{aligned}
$$

where $\lambda_{1}, \ldots, \lambda_{n}$ are the eigenvalues of $A$. So every entry of $(I-z A)^{-1}$ takes the form

$$
\begin{aligned}
\left(p_{0}+p_{1} z+\cdots\right. & \left.+p_{m} z^{m}\right) \prod_{j=1}^{n} \sum_{i=0}^{\infty} \lambda_{j}^{i} z^{i} \\
& =\sum_{k=0}^{\infty} \sum_{u=0}^{m} p_{u} \sum_{i_{1}+i_{2}+\cdots+i_{n}=k-u} \lambda_{1}^{i_{1}} \lambda_{2}^{i_{2}} \cdots \lambda_{n}^{i_{n}} z^{k} .
\end{aligned}
$$

Since the eigenvalues of a complex matrix vary continuously with entries, the lemma follows.

Now let $S(n)$ denote the set of all the $n \times n$ complex matrices with isolated (in modulus) maximum eigenvalue.

Lemma 7.6: $S(n)$ is connected.

Proof: Let $A, B \in S(n)$, then we consider their Jordan forms

$$
\begin{aligned}
& A=U \operatorname{diag}\left(\lambda_{1}, C\right) U^{-1} \\
& B=V \operatorname{diag}\left(\eta_{1}, D\right) V^{-1}
\end{aligned}
$$

here $\lambda_{1}, \eta_{1}$ are maximum eigenvalues for $A, B$, respectively, $C, D$ correspond to other Jordan blocks, and $U, V \in G L(n, \mathbb{C})$ (here $G L(n, \mathbb{C})$ denotes the set of all the $n \times n$ nonsingular complex matrices). Since $G L(n, \mathbb{C})$ is connected [19], it suffices to prove that there is a path in $S(n)$ from $\operatorname{diag}\left(\lambda_{1}, C\right)$ to $\operatorname{diag}\left(\eta_{1}, D\right)$. This is straightforward: first connect $\operatorname{diag}\left(\lambda_{1}, C\right)$ to $\operatorname{diag}\left(\eta_{1}, \eta_{1} / \lambda_{1} C\right)$ by a continuous rescaling; then connect $\eta_{1} / \lambda_{1} C$ to $D$ by the path $t \eta_{1} / \lambda_{1} C+(1-t) D$ (the path $\operatorname{diag}\left(\eta_{1}, t \eta_{1} / \lambda_{1} C+(1-t) D\right)$ stays within $S(n)$ since the matrices along this path are upper triangular with all diagonal entries, except $\eta_{1}$, of modulus less than $\left|\eta_{1}\right|$ ).

For a complex analytic function $f\left(z_{1}, z_{2}, \ldots, z_{n}\right)$, let $V(f)$ denote the "hypersurface" defined by $f$, namely

$$
V(f)=\left\{\left(z_{1}, z_{2}, \ldots, z_{n}\right) \in \mathbb{C}^{n}: f\left(z_{1}, z_{2}, \ldots, z_{n}\right)=0\right\} .
$$

Now let $\Omega_{\mathbb{C}}$ denote a connected open set in $\mathbb{C}^{n}$. It is well known that the following lemma holds (for completeness, we include a brief proof).

Lemma 7.7: $\Omega_{\mathbb{C}} \backslash V(f)$ is connected.

Proof: For simplicity, we first assume $\Omega_{\mathbb{C}}$ is a ball $B_{r}\left(z_{0}\right)$ (here $z_{0} \in \mathbb{C}^{n}$ is the center of the ball and $r$ is the radius, i.e., $\left.B_{r}\left(z_{0}\right)=\left\{z \in \mathbb{C}^{n}:\left|z-z_{0}\right|<r\right\}\right)$ in $\mathbb{C}^{n}$. For any two distinct points $P, Q \in \Omega_{\mathbb{C}} \backslash V(f)$, consider the "complex line"

$$
L_{\mathbb{C}}^{P Q}=\{z P+(1-z) Q: z \in \mathbb{C}\} .
$$

$L_{\mathbb{C}}^{P Q} \cap V(f) \cap \Omega_{\mathbb{C}}$ consists of only isolated points (A nonconstant one variable complex analytic function must have isolated zeros in the complex plane [30]). It then follows that for the compact real line segment

$$
L_{\mathbb{R}}^{P Q}=\{t P+(1-t) Q: t \in[0,1]\}
$$

$L_{\mathbb{R}}^{P Q} \cap V(f) \cap \Omega_{\mathbb{C}}$ consists of only finitely many points. Certainly one can choose an arc in $L_{\mathbb{C}}^{P Q} \cap \Omega_{\mathbb{C}}$ to avoid these points and connect $P$ and $Q$. This implies that $\Omega_{\mathbb{C}} \backslash V(f)$ is connected.

In the general case, $\Omega_{\mathbb{C}}$ is a connected open set in $\mathbb{C}^{n}$. Let $I$ be an arc in $\Omega_{\mathbb{C}}$ connecting $P$ and $Q$, and let $\left\{B_{r_{j}}\left(z_{j}\right)\right\}$ be a collection of balls covering $I$ such that each $B_{r_{j}}\left(z_{j}\right) \cap B_{r_{j+1}}\left(z_{j+1}\right) \neq$ $\phi$. Pick a point $P_{j}$ in $B_{r_{j}}\left(z_{j}\right) \cap B_{r_{j+1}}\left(z_{j+1}\right)$ such that $P_{j} \in$ $\Omega_{\mathbb{C}} \backslash V(f)$. Applying the same argument as above to every ball $B_{r_{j}}\left(z_{j}\right)$, we see that $P$ is connected to $Q$ in $\Omega_{\mathbb{C}} \backslash V(f)$ through the points $P_{j}$ 's. Thus, we prove the lemma.

Theorem 7.8: Let $\Delta$ be an irreducible stochastic $d \times d$ matrix. Write $\Delta$ in the form

$$
\Delta=\left[\begin{array}{ll}
a & r \\
c & B
\end{array}\right]
$$

where $a$ is a scalar and $B$ is a $(d-1) \times(d-1)$ matrix. Let $\Phi$ be the function defined by $\Phi(1)=0$, and $\Phi(2)=\cdots=\Phi(d)=$ 1 . Then for any parametrization $\Delta(\vec{\varepsilon})$ such that $\Delta\left(\vec{\varepsilon}_{0}\right)=\Delta$, letting $Z^{\vec{\varepsilon}}$ denote the hidden Markov chain defined by $\Delta(\vec{\varepsilon})$ and $\Phi, H\left(Z^{\vec{\varepsilon}}\right)$ is analytic at $\vec{\varepsilon}_{0}$ if and only if

1. $a>0$, and $r B^{j} c>0$ for $j=0,1, \cdots$;

2. the maximum eigenvalue of $B$ is simple and strictly greater in absolute value than the other eigenvalues of $B$.

Proof:

Proof of sufficiency. We write

$$
\Delta(\vec{\varepsilon})=\left[\begin{array}{ll}
a(\vec{\varepsilon}) & r(\vec{\varepsilon}) \\
c(\vec{\varepsilon}) & B(\vec{\varepsilon})
\end{array}\right]
$$

where $a(\vec{\varepsilon})$ is a scalar and $B(\vec{\varepsilon})$ is a $(d-1) \times(d-1)$ matrix.

Since $\Delta\left(\vec{\varepsilon}_{0}\right)$ is stochastic and irreducible, its spectral radius is 1 , and 1 is a simple eigenvalue of $\Delta$. Thus, if $\Omega_{\mathbb{C}}$ is sufficiently small, for all $\vec{\varepsilon} \in \Omega_{\mathbb{C}}$, any fixed row $\pi(\vec{\varepsilon})=\left(\pi_{1}(\vec{\varepsilon}), \pi_{2}(\vec{\varepsilon}), \ldots, \pi_{d}(\vec{\varepsilon})\right)$ of $\operatorname{Adj}(I-\Delta(\vec{\varepsilon}))$ is a left eigenvector of $\Delta(\vec{\varepsilon})$ associated with eigenvalue 1 and is an analytic function of $\vec{\varepsilon}$. Normalizing, we can assume that $\pi(\vec{\varepsilon}) \mathbf{1}=1, \pi(\vec{\varepsilon})$ is analytic in $\vec{\varepsilon}$, and $\pi_{j}\left(\vec{\varepsilon}_{0}\right)>0$ for $j=1,2, \ldots, d$. 
The entries of $r(\vec{\varepsilon}), B(\vec{\varepsilon})$, and $c(\vec{\varepsilon})$ are real analytic in $\vec{\varepsilon}$ and can be extended to complex analytic functions in a complex neighborhood $\Omega_{\mathbb{C}}$ of $\vec{\varepsilon}_{0}$. Thus, for all $n$

$$
\pi_{1}(\vec{\varepsilon}) r(\vec{\varepsilon}) B(\vec{\varepsilon})^{n-1} \mathbf{1} \quad \text { and } \quad \pi_{1}(\vec{\varepsilon}) r(\vec{\varepsilon}) B(\vec{\varepsilon})^{n-1} c(\vec{\varepsilon})
$$

can be extended to complex analytic functions on $\Omega_{\mathbb{C}}$ (in fact, each of these functions is a polynomial in $\vec{\varepsilon}$ ).

Since $B\left(\vec{\varepsilon}_{0}\right)$ is a proper submatrix of the irreducible stochastic matrix $\Delta\left(\vec{\varepsilon}_{0}\right)$, its spectral radius is strictly less than 1 . Thus, by Lemma 7.5, there exists $0<\lambda^{*}<1$ and a constant $C_{1}>0$, such that for some complex neighborhood $\Omega_{\mathbb{C}}$ of $\vec{\varepsilon}_{0}$, all $\vec{\varepsilon} \in \Omega_{\mathbb{C}}$, and all $n$

$$
\left|B_{i j}^{n}(\vec{\varepsilon})\right|<C_{1}\left(\lambda^{*}\right)^{n} .
$$

Since $\pi_{1}(\vec{\varepsilon}), r(\vec{\varepsilon})$ and $c(\vec{\varepsilon})$ are continuous in $\vec{\varepsilon}$, there is a constant $C_{2}>0$ such that for all $\vec{\varepsilon} \in \Omega_{\mathbb{C}}$ and all $n$

$$
\left|\pi_{1}(\vec{\varepsilon}) r(\vec{\varepsilon}) B(\vec{\varepsilon})^{n} 1\right|<C_{2}\left(\lambda^{*}\right)^{n} .
$$

We will need the following result, proven in Appendix B.

Lemma 7.9: Let

$$
\begin{aligned}
a(\vec{\varepsilon}, n) & \equiv \frac{\pi_{1}(\vec{\varepsilon}) r(\vec{\varepsilon}) B(\vec{\varepsilon})^{n} \mathbf{1}}{\pi_{1}(\vec{\varepsilon}) r(\vec{\varepsilon}) B(\vec{\varepsilon})^{n-1} \mathbf{1}} \\
b(\vec{\varepsilon}, n) & \equiv \frac{\pi_{1}(\vec{\varepsilon}) r(\vec{\varepsilon}) B(\vec{\varepsilon})^{n-1} c(\vec{\varepsilon})}{\pi_{1}(\vec{\varepsilon}) r(\vec{\varepsilon}) B(\vec{\varepsilon})^{\frac{n-1}{1}}} .
\end{aligned}
$$

For a sufficiently small neighborhood $\Omega_{\mathbb{C}}$ of $\vec{\varepsilon}_{0}$, both $|a(\vec{\varepsilon}, n)|$ and $|b(\vec{\varepsilon}, n)|$ are bounded from above and away from zero, uniformly in $\vec{\varepsilon} \in \Omega_{\mathbb{C}}$ and $n$.

Define

$$
H_{n}^{\vec{\varepsilon}}=-a(\vec{\varepsilon}, n) \log a(\vec{\varepsilon}, n)-b(\vec{\varepsilon}, n) \log b(\vec{\varepsilon}, n)
$$

where $a(\vec{\varepsilon}, n)$ and $b(\vec{\varepsilon}, n)$ are as in Lemma 7.9. Choosing $\Omega_{\mathbb{C}}$ to be a smaller neighborhood of $\vec{\varepsilon}_{0}$, if necessary, $a(\vec{\varepsilon}, n)$ and $b(\vec{\varepsilon}, n)$ are constrained to lie in a closed disk not containing 0 . Thus, for all $n, H_{n}^{\vec{\varepsilon}}$ is an analytic function of $\vec{\varepsilon}$, with $\left|H_{n}^{\vec{\varepsilon}}\right|$ bounded uniformly in $\vec{\varepsilon} \in \Omega_{\mathbb{C}}$ and $n$. Since $\pi_{1}(\vec{\varepsilon}) r(\vec{\varepsilon}) B(\vec{\varepsilon})^{n-1} \mathbf{1}$ is analytic on $\Omega_{\mathbb{C}}$ and exponentially decaying (by (7.26)), the infinite series

$$
\begin{aligned}
H^{\vec{\varepsilon}}(Z)=\pi_{1}(\vec{\varepsilon}) H_{0}^{\vec{\varepsilon}} & +\pi_{1}(\vec{\varepsilon}) r(\vec{\varepsilon}) 1 H_{1}^{\vec{\varepsilon}}+\cdots \\
& +\pi_{1}(\vec{\varepsilon}) r(\vec{\varepsilon}) B(\vec{\varepsilon})^{n-1} 1 H_{n}^{\vec{\varepsilon}}+\cdots
\end{aligned}
$$

converges uniformly on $\Omega_{\mathbb{C}}$ and thus defines an analytic function on $\Omega_{\mathbb{C}}$.

Note that for $\vec{\varepsilon} \geq 0$

$$
p^{\vec{\varepsilon}}\left(1^{(n)} 0\right)=\pi_{1}(\vec{\varepsilon}) r(\vec{\varepsilon}) B(\vec{\varepsilon})^{n-1} \mathbf{1}
$$

and

$$
p^{\vec{\varepsilon}}\left(01^{(n)} 0\right)=\pi_{1}(\vec{\varepsilon}) r(\vec{\varepsilon}) B(\vec{\varepsilon})^{n-1} c(\vec{\varepsilon}) .
$$

By (7.28), (7.29), and (7.22), $H^{\vec{\varepsilon}}(Z)$ agrees with the entropy rate when $\Delta(\vec{\varepsilon}) \geq 0$, as desired.

Remark 7.10: We show how sufficiency relates to Theorem 6.1. Namely, the assumptions in Theorem 7.8 imply those of
Theorem 6.1. Condition 1 of Theorem 6.1 follows from the fact that $\Delta$ is assumed irreducible. For conditions 2 and 3 of Theorem 6.1, one first notes that the image of $f_{0}$ is a single point $W_{0}$, and the $f_{1}$-orbit of $W_{0}$ and $f_{1}$-orbit of $\vec{s}$ converge to a point $p_{1}$. It follows that $L$ is the union of $W_{0}$, the $f_{1}$-orbit of $W_{0}$ and $p_{1}$. The assumptions in Theorem 7.8. imply that $r_{a}>0$ on $L$ (i.e., condition 2 of Theorem 6.1 holds) and that for sufficiently large $n$, the $n$-fold composition of $f_{1}$ is contracting on the convex hull of the intersection of $L$ and $W_{1}$ (so condition 3 of Theorem 6.1 holds). To see the latter, one uses the ideas in the proof of sufficiency.

\section{Proof:}

Proof of necessity We first consider Condition 2. We shall use the natural parameterization and view $H(Z)$ as a function of $\Delta$, or more precisely of $(B, r)$. Note that there is a one-to-one correspondence between $\Delta$ and $(B, r)$; we shall use this correspondence throughout the proof.

Suppose $\Delta$ does not satisfy Condition 2, however, $H(Z)$ is analytic at $\Delta$ with respect to the natural parameterization. In other words, suppose there exists a complex neighborhood $N_{\Delta}$ of $\Delta$ (here $N_{\Delta}$ corresponds to $N_{B} \times N_{r}$ where $N_{B}$ is neighborhood of $B$ and $N_{r}$ is neighborhood of $r$ ) such that $H(Z)$ can be analytically extended to $N_{\Delta}$, while the corresponding $B$ does not have isolated (in modulus) maximum eigenvalue.

We first claim there exists $\tilde{\Delta} \in N_{\Delta}$ with $\tilde{r} \tilde{B}^{k} 1=0$, here $\tilde{r}$ and $\tilde{B}$ correspond to $\tilde{\Delta}$ and $\tilde{B}$ has distinct eigenvalues (in modulus). Indeed, we can first (for simplicity) perturb $\Delta$ to $\tilde{\Delta}$ such that the corresponding $\tilde{B}$ has distinct eigenvalues in modulus. Then

$$
\begin{aligned}
\tilde{B}= & \tilde{U} \operatorname{diag}\left(\tilde{\lambda}_{1}, \tilde{\lambda}_{2}, \ldots, \tilde{\lambda}_{d-1}\right) \tilde{U}^{-1} \\
= & \left(\tilde{v}_{1}, \tilde{v}_{2}, \ldots, \tilde{v}_{d-1}\right) \operatorname{diag}\left(\tilde{\lambda}_{1}, \tilde{\lambda}_{2}, \ldots, \tilde{\lambda}_{d-1}\right) \\
& \times\left(\tilde{w}_{1}^{t}, \tilde{w}_{2}^{t}, \ldots, \tilde{w}_{d-1}^{t}\right)^{t}
\end{aligned}
$$

where $\left|\tilde{\lambda}_{1}\right|>\left|\tilde{\lambda}_{2}\right|>\cdots>\left|\tilde{\lambda}_{d-1}\right|$, and $\tilde{v}_{i}, \tilde{w}_{i}$ 's are appropriately scaled right and left eigenvectors of $\tilde{B}$, respectively. Then we have

$$
r \tilde{B}^{k} \mathbf{1}=r \tilde{v}_{1} \tilde{w}_{1} \mathbf{1} \tilde{\lambda}_{1}^{k}+r \tilde{v}_{2} \tilde{w}_{2} \mathbf{1} \tilde{\lambda}_{2}^{k}+\cdots+r \tilde{v}_{d-1} \tilde{w}_{d-1} \mathbf{1} \tilde{\lambda}_{d-1}^{k} .
$$

Further, consider a perturbation of $B$ from

$$
\tilde{B}=\tilde{U} \operatorname{diag}\left(\tilde{\lambda}_{1}, \tilde{\lambda}_{2}, \ldots, \tilde{\lambda}_{d-1}\right) \tilde{U}^{-1}
$$

to

$$
\tilde{B}=V \tilde{U} \operatorname{diag}\left(\tilde{\lambda}_{1}, \tilde{\lambda}_{2}, \ldots, \tilde{\lambda}_{d-1}\right) \tilde{U}^{-1} V^{-1}
$$

where $V$ is a complex matrix close to the $(d-1) \times(d-1)$ identity matrix $I_{d-1}$. So we can pick $V$ such that $\tilde{v}_{1} \tilde{w}_{1} V^{-1} 1 \neq 0$, $\tilde{v}_{1} \tilde{w}_{1} V^{-1} \tilde{c} \neq 0, \tilde{v}_{2} \tilde{w}_{2} V^{-1} 1 \neq 0$. Clearly, $\tilde{v}_{1} \tilde{w}_{1} V^{-1} 1$ is not proportional to $\tilde{v}_{2} \tilde{w}_{2} V^{-1} 1$. Then by a further perturbation of $r$ to $\tilde{r}$, we can simultaneously require that $\tilde{r} \tilde{v}_{1} \tilde{w}_{1} \mathbf{1} \neq 0, \tilde{r} \tilde{v}_{1} \tilde{w}_{1} \tilde{c} \neq$ $0, \tilde{r} \tilde{v}_{2} \tilde{w}_{2} \mathbf{1} \neq 0,\left|\tilde{r} \tilde{v}_{1} \tilde{w}_{1} \mathbf{1}\right| \neq\left|\tilde{r} \tilde{v}_{2} \tilde{w}_{2} \mathbf{1}\right|$, where we redefine $\tilde{v}_{i}=V \tilde{v}_{i}$ and $\tilde{w}_{i}=\tilde{w}_{i} V^{-1}$. For any $\theta$ and $\eta>0$, it can be checked that

$$
\bigcup_{k=0}^{\infty}\left\{z^{k}:\left|z-e^{i \theta}\right|<\eta\right\}=\mathbb{C} \backslash\{0\} .
$$


Since $\tilde{\lambda}_{2}$ is a perturbation of $\tilde{\lambda}_{1}$, it follows that for large enough $k$, one can perturb $\tilde{\lambda}_{2}$ to satisfy the equation at the bottom of the page, with $\left|\tilde{\lambda}_{2}\right| \neq\left|\tilde{\lambda}_{1}\right|$ and $\left|\tilde{\lambda}_{2}\right|$ strictly greater than $\left|\tilde{\lambda}_{j}\right|$ for $j \geq 3$. Thus we prove the claim.

We now pick a positive matrix $\hat{\Delta} \in N_{\Delta}$ with corresponding $\hat{r}$ and $\hat{B}$. We then pick $\tilde{\Delta} \in N_{\Delta}$ with corresponding $\tilde{r}$ and $\tilde{B}$ (with distinct eigenvalues in modulus) such that $\tilde{r} \tilde{B}^{k_{1}} \mathbf{1}=0$ for some $k_{1}$, and we can further require that $\tilde{r} \tilde{v}_{1} \tilde{w}_{1} \mathbf{1} \neq 0, \tilde{r} \tilde{v}_{1} \tilde{w}_{1} \tilde{c} \neq 0$ (see the proof for the previous claim), where as before, $\tilde{v}_{1}, \tilde{w}_{1}$ are eigenvectors corresponding to the largest eigenvalue of $\tilde{B}$. According to Lemma 7.6, there is an arc $I_{1} \subset S(d-1)$ connecting $\hat{B}$ to $\tilde{B}$; we then connect $\hat{r}$ and $\tilde{r}$ using an arc $I_{2}$ in $\mathbb{C}^{d-1}$. According to Lemma 7.7, we can choose the $\operatorname{arc} I=\left(I_{1}, I_{2}\right)$ to avoid the hypersurface

$$
V\left(\left(r v_{1} w_{1} \mathbf{1}\right)\left(r v_{1} w_{1} c\right)\right) \subset \mathbb{C}^{(d-1)^{2}} \times \mathbb{C}^{d-1} ;
$$

in other words, we can assume that along the path $I, r v_{1} w_{1} \mathbf{1} \neq$ 0 and $r v_{1} w_{1} c \neq 0$; here $v_{1}, w_{1}, c$ are determined by the variable matrix $B$ along the path $I_{1}$ and $r$ is the variable point along path $I_{2}$ (we remind the reader that the coordinates of $v_{1}$ and $w_{1}$ are all analytic functions of the entries of $B$ ). We then claim that there is a neighborhood $N_{I}$ of $I$ such that $V_{k} \cap N_{I} \neq \phi$ and $W_{k} \cap N_{I} \neq \phi$ hold for only finitely many $k$, where $V_{k}=$ $\left\{(B, r): r B^{k} 1=0\right\}$ and $W_{k}=\left\{(B, r): r B^{k} c=0\right\}$. Indeed, for any $\Delta \in I$ with corresponding $B \in S(d-1)$, by the Jordan form we have

$$
r B^{k} \mathbf{1}=r v_{1} w_{1} \mathbf{1} \lambda_{1}^{k}+o\left(\lambda_{1}^{k}\right)
$$

where $\lambda_{1}$ is the isolated maximum eigenvalue and $v_{1}, w_{1}$ are appropriately scaled right and left eigenvectors of $B$, respectively. Since $r v_{1} w_{1} \mathbf{1} \neq 0$ on $I$, there exists a complex connected neighborhood $N_{I}$ of $I$ such that $r v_{1} w_{1} 1 \neq 0$ on $N_{I}$ and $r v_{1} w_{1} 1 \lambda_{1}^{k}$ dominates uniformly on $N_{I}$ (see Lemma 7.5). Consequently, $\left|r B^{k} \mathbf{1}\right|>0$ on $N_{I}$ for large enough $k$. In other words, $V_{k} \cap N_{I} \neq \phi$ holds for only finitely many $k$. Similarly, since $r v_{1} w_{1} c \neq 0$ on $I$, there exists a complex neighborhood $N_{I}$ of $I$ (here we use the same notation for a possibly different neighborhood) such that $W_{k} \cap N_{I} \neq \phi$ holds only for finitely many $k$. From now on, we assume such $k$ 's are less than some $K$, which depends on $N_{I}$.

We claim that we can further choose $I$ and find a new neighborhood $N_{I}$ in $\mathbb{C}^{d-1} \times S(d-1)$ of $I$ such that $V_{k} \cap N_{I} \neq \phi$ holds only for $k=k_{1}$ and $W_{k} \cap N_{I}=\phi$ for all $k$. Consider $\tilde{\Delta}$ with corresponding $\tilde{B}$, let $F_{i}=F_{i}(\tilde{B})=\left\{r: r \tilde{B}^{i} 1=0\right\}$, which is a hyperplane orthogonal to the vector $\tilde{B}^{i} 1$ in $\mathbb{C}^{d-1}$. Similarly, we define $G_{i}=G_{i}(\tilde{B})=\left\{r: r \tilde{B}^{i} \tilde{c}=0\right\}$. Recall that $\tilde{B}=\tilde{U} \operatorname{diag}\left(\lambda_{1}, \lambda_{2}, \ldots, \lambda_{d-1}\right) \tilde{U}^{-1}$; we can require that $\tilde{U}^{-1} 1$ has no zero coordinates by a small perturbation of $\tilde{U}$ if necessary. We then show that $F_{i}$ 's and $G_{j}$ 's define different hyperplanes in $\mathbb{C}^{d-1}$. Indeed, suppose $F_{i}=F_{j}$.
It follows that $\tilde{U} \operatorname{diag}\left(\tilde{\lambda}_{1}^{i}, \tilde{\lambda}_{2}^{i}, \ldots, \tilde{\lambda}_{d-1}^{i}\right) \tilde{U}^{-1} \mathbf{1}$ is proportional to $\tilde{U} \operatorname{diag}\left(\tilde{\lambda}_{1}^{j}, \tilde{\lambda}_{2}^{j}, \ldots, \tilde{\lambda}_{d-1}^{j}\right) \tilde{U}^{-1} 1$. It then follows that $\left(\tilde{\lambda}_{1}^{i}, \tilde{\lambda}_{2}^{i}, \ldots, \tilde{\lambda}_{d-1}^{i}\right)$ is proportional to $\left(\tilde{\lambda}_{1}^{j}, \tilde{\lambda}_{2}^{j}, \ldots, \tilde{\lambda}_{d-1}^{j}\right)$. However, since not all eigenvalues have the same modulus, this implies that $i=j$. With a perturbation of $\tilde{c}$ (equivalently, a perturbation of row sums of $\tilde{B}$ ), if necessary, we conclude that the $F_{i}$ 's and $G_{i}$ 's determine different hyperplanes, i.e., $F_{i} \neq F_{j}, G_{i} \neq G_{j}$ for $i \neq j \leq K$, and $F_{i} \neq G_{j}$ for all $i, j$. Thus, with a perturbation of $\tilde{r}$ if necessary, we can choose a new $\tilde{\Delta}$ contained in $V_{k_{1}}$, but not contained in any $V_{k}$ with $k \neq k_{1}$ or $W_{k}$ for all $k$. Again, by Lemma 7.7, one can choose a new $I$ inside original $N_{I}$, connecting $\hat{\Delta}$ and $\tilde{\Delta}$, to avoid all $V_{k}$ 's and $W_{k}$ 's except $V_{k_{1}}$, then choose a smaller new neighborhood $N_{I}$ of the new $I$ to make sure that $V_{k} \cap N_{I} \neq \phi$ only holds for $k=k_{1}$ and $W_{k} \cap N_{I}=\phi$ for all $k$.

Since the perturbed complex matrix $B$ still has spectral radius strictly less than 1 , all the complexified terms in the entropy rate formula (see (7.27)) with $k \neq k_{1}$ are exponentially decaying and thus sum up to an analytic function on $N_{I}$ (i.e., the sum of these terms can be analytically continued to $N_{I}$ ), while the unique analytic extension of the $k_{1}$ th term on $N_{I}$ blows up as one approaches $V_{k_{1}} \cap N_{I}$ from $\hat{\Delta}$. Again, by the uniqueness of analytic extension of $H(Z)$ on $N_{I}$, this would be a contradiction to the assumption that $H(Z)$ is analytic at $\Delta$ (here we are applying the uniqueness theorem of analytic continuation of a function of several complex variables, see [30, p. 21]). Thus, we prove the necessity of Condition 2.

We now consider Condition 1. Suppose $\Delta$ does not satisfies Condition 1, namely, $a=0$ or $r B^{k} c=0$ for some $k$, however, $H(Z)$ is analytic at $\Delta$. With the proof above for the necessity of Condition 2, we can now assume the corresponding $B \in$ $S(d-1)$.

If $a=0$, consider any perturbation of $\Delta$ to $\Delta_{1}$ such that $\tilde{B} \in S(d-1), \tilde{r} \tilde{v}_{1} \tilde{w}_{1} \mathbf{1} \neq 0, \tilde{r} \tilde{v}_{1} \tilde{w}_{1} \tilde{c} \neq 0, \tilde{r} \tilde{B}^{k} \mathbf{1} \neq 0$ and $\tilde{r} \tilde{B}^{k} \tilde{c} \neq 0$ for all $k$ (here we follow the notation as in the proof of necessity of Condition 2). Then using similar arguments, we can prove the sum of all the terms except the first term in the entropy rate formula (see (7.27)) can be analytically extended to $\tilde{\Delta}$. However, this implies that $a \log a$ is a well-defined analytic function on some neighborhood of 0 in $\mathbb{C}$, which is a contradiction. Similar arguments can be applied to the case that $r B^{k} c=0$ for some $k$ 's. Thus, we prove the necessity of Condition 1 .

\section{ANALYTicity OF A HidDEN Markov Chain IN A STRONG SENSE}

In this section, we show that if $\Delta$ is analytically parameterized by a real variable vector $\vec{\varepsilon}$, and at $\vec{\varepsilon}_{0}, \Delta$ satisfies Conditions 1 and 2 of Theorem 1.1, then the hidden Markov chain itself is a real analytic function of $\vec{\varepsilon}$ at $\vec{\varepsilon}_{0}$ in a strong sense. We assume (for this section only) that the reader is familiar with the basics of measure theory and functional analysis [17], [34], [18]. Our

$$
\left(\tilde{\lambda}_{2} / \tilde{\lambda}_{1}\right)^{k}=\frac{-\tilde{r} \tilde{v}_{1} \tilde{w}_{1} \mathbf{1}-\tilde{r} \tilde{v}_{3} \tilde{w}_{3} \mathbf{1}\left(\tilde{\lambda}_{3} / \tilde{\lambda}_{1}\right)^{k}-\cdots-\tilde{r} \tilde{v}_{d-1} \tilde{w}_{d-1} \mathbf{1}\left(\tilde{\lambda}_{d-1} / \tilde{\lambda}_{1}\right)^{k}}{\tilde{r} \tilde{v}_{2} \tilde{w}_{2} \mathbf{1}}
$$


approach uses a connection between the entropy rate of a hidden Markov chain and symbolic dynamics explored in [16].

Let $\mathcal{X}$ denote the set of left infinite sequences with finite alphabet. A cylinder set is a set of the form $\left(\left\{x_{-\infty}^{0}: x_{0}=\right.\right.$ $\left.\left.z_{0}, \ldots, x_{-n}=z_{-n}\right\}\right)$. The Borel sigma-algebra is the smallest sigma-algebra containing the cylinder sets. A Borel probability measure $(\mathrm{BPM}) \nu$ on $\mathcal{X}$ is a measure on the Borel measurable sets of $\mathcal{X}$ such that $\nu(\mathcal{X})=1$. Such a measure is uniquely determined by its values on the cylinder sets.

For real $\vec{\varepsilon}$, consider the measure $\nu^{\vec{\varepsilon}}$ on $\mathcal{X}$ defined by

$$
\nu^{\vec{\varepsilon}}\left(\left\{x_{-\infty}^{0}: x_{0}=z_{0}, \ldots, x_{-n}=z_{-n}\right\}\right)=p^{\vec{\varepsilon}}\left(z_{-n}^{0}\right) .
$$

Note that $H(Z)$ can be rewritten as

$$
H^{\vec{\varepsilon}}(Z)=\int-\log p^{\vec{\varepsilon}}\left(z_{0} \mid z_{-\infty}^{-1}\right) d \nu^{\vec{\varepsilon}} .
$$

Usually, the Borel sigma-algebra is defined to be the smallest sigma-algebra containing the open sets; in this case, the open sets are defined by the metric: for any two elements $\xi$ and $\eta$ in $\mathcal{X}$, define $d(\xi, \eta)=2^{-k}$ where $k=\inf \left\{|i|: \xi_{i} \neq \eta_{i}\right\}$. The metric space $(\mathcal{X}, d)$ is compact.

Let $C(\mathcal{X})$ be the space of real-valued continuous functions on $\mathcal{X}$. Then $C(\mathcal{X})$ is a Banach space (i.e., complete normed linear space) with the sup norm $\|f\|_{\infty}=\sup \{|f(x)|: x \in \mathcal{X}\}$. Then any $\operatorname{BPM} \nu$ acts as a bounded linear functional on $C(\mathcal{X})$, namely, $\nu(f)=\int f d \nu$. As such, the set of BPMs is a subset of the dual space $C(\mathcal{X})^{*}$, which is itself a Banach space; the norm of a $\operatorname{BPM} \nu$ is defined as $\|\nu\|=\sup _{\left\{f \in C(\mathcal{X}):\|f\|_{\infty}=1\right\}} \int f d \nu$. In fact, since $\mathcal{X}$ is compact, $C(\mathcal{X})^{*}$ is the linear span of the BPMs.

It makes sense to ask if $\vec{\varepsilon} \mapsto \nu^{\vec{\varepsilon}}$ is analytic as a mapping from the parameter space to $C(\mathcal{X})^{*}$; by definition, this would mean that $\nu^{\vec{\varepsilon}}$ can be expressed as a power series in the coordinates of $\vec{\varepsilon}$. However, as the following example shows, this mapping is not even continuous.

Let $\mathcal{X}$ be the set of binary left infinite sequences. Let $\nu_{p}$ denote the i.i.d. $(p, 1-p)$ measure, with $0<p<1$. We claim that, for fixed $p>q$ and $\varepsilon$ with $0<\varepsilon<(p-q) / 2$, by application of the law of large numbers to $\nu_{p}$ and $\nu_{q}$, one can find a finite union $C$ of cylinder sets such that

$$
\nu_{p}(C)>1-\varepsilon
$$

and

$$
\nu_{q}(C)<\varepsilon
$$

To see this, first say that a word $w=w_{1} \ldots w_{n}$ is $(p, \varepsilon, n)$ typical if the frequency of 0 's in $w$ is in $[p-\varepsilon, p+\varepsilon]$. Let $C_{p, \varepsilon, n}$ be the union of the cylinder sets corresponding to the $(p, \varepsilon, n)$-typical words. The law of large numbers asserts that for $n$ sufficiently large

$$
\nu_{p}\left(C_{p, \varepsilon, n}\right)>1-\varepsilon \quad \text { and } \quad \nu_{q}\left(C_{q, \varepsilon, n}\right)>1-\varepsilon .
$$

Since $p>q+2 \varepsilon$, then $C_{p, \varepsilon, n}$ and $C_{q, \varepsilon, n}$ are disjoint. Thus, we have

$$
\nu_{q}\left(C_{p, \varepsilon, n}\right)<1-\nu_{q}\left(C_{q, \varepsilon, n}\right)<\varepsilon .
$$

Set $C=C_{p, \varepsilon, n}$.
Now, if $f$ is the characteristic function of $C$, we have

$\left\|\nu_{p}-\nu_{q}\right\| \geq \int f d \nu_{p}-\int f d \nu_{q}=\nu_{p}(C)-\nu_{q}(C)>1-2 \varepsilon$.

So, $\left\|\nu_{p}-\nu_{q}\right\| \geq 1$. It follows that $\nu_{q}$ cannot converge in norm to $\nu_{p}$ as $q \rightarrow p$, and so the map $p \mapsto \nu_{p}$ from $R$ to $C(\mathcal{X})^{*}$ is discontinuous.

On the other hand, using the work of Ruelle [26], we now show that $\vec{\varepsilon} \mapsto \nu^{\vec{\varepsilon}}$ is analytic as a mapping from the parameter space to another natural space.

For $f \in C(\mathcal{X})$, define

$$
\operatorname{var}_{n}(f)=\sup \left\{\left|f(\xi)-f\left(\xi^{\prime}\right)\right|: \xi_{-i}=\xi_{-i}^{\prime} \text { for } i \leq n\right\} .
$$

We denote by $F^{\theta}$ the subset of $f \in C(\mathcal{X})$ such that

$$
\|f\|_{\theta} \equiv \sup _{n \geq 0}\left(\theta^{-n} \operatorname{var}_{n}(f)\right)<+\infty .
$$

$F^{\theta}$ is a Banach space with the norm $\|f\|=\max \left(\|f\|_{\infty},\|f\|_{\theta}\right)$. Let $\left(F^{\theta}\right)^{*}$ denote the dual space (i.e., the set of bounded linear functionals) on $F^{\theta}$. For any $\nu \in\left(F^{\theta}\right)^{*}$, the norm of $\nu$ is naturally defined as $\|\nu\|=\sup _{\left\{f \in F^{\theta}:\|f\|=1\right\}} \nu(f)$. Using complex functions instead of real functions, one defines $F_{\mathbb{C}}^{\theta}$ and $\left(F_{\mathbb{C}}^{\theta}\right)^{*}$ similarly.

In the following theorem, we prove the analyticity of a hidden Markov chain in a strong sense.

Theorem 8.1: Suppose that the entries of $\Delta$ are analytically parameterized by a real variable vector $\vec{\varepsilon}$. If at $\vec{\varepsilon}=\vec{\varepsilon}_{0}, \Delta$ satisfies Conditions 1 and 2 in Theorem 1.1, then the mapping $\vec{\varepsilon} \mapsto \log p^{\vec{\varepsilon}}\left(z_{0} \mid z_{-\infty}^{-1}\right)$ is analytic at $\vec{\varepsilon}_{0}$ from the real parameter space to $F^{\rho}$ (here $\rho$ is the contraction constant in the proof of Theorem 1.1). Moreover, the mapping $\vec{\varepsilon} \mapsto \nu^{\vec{\varepsilon}}$ is analytic at $\vec{\varepsilon}_{0}$ from the real parameter space to $\left(F^{\rho}\right)^{*}$.

Proof: For complex $\vec{\varepsilon}$, by (4.16), one shows that $\log p^{\vec{\varepsilon}}\left(z_{0} \mid z_{-\infty}^{-1}\right)$ can be defined on $\Omega_{\mathbb{C}}$ as the uniform (in $\vec{\varepsilon}$ and $z \in \mathcal{X}$ ) limit of $\log p^{\vec{\varepsilon}}\left(z_{0} \mid z_{-n}^{-1}\right)$ as $n \rightarrow \infty$, and $\log p^{\vec{\varepsilon}}\left(z_{0} \mid z_{-\infty}^{-1}\right)$ belongs to $F_{\mathbb{C}}^{\rho}$. By (4.5), (4.6), (4.7), and (4.14) it follows that $p^{\vec{\varepsilon}}\left(z_{0} \mid z_{-n}^{-1}\right)$ is analytic on $\Omega_{\mathbb{C}}$. As a result of $(4.16)$, if $\Delta$ satisfies Conditions 1 and 2, for fixed $z \in \mathcal{X}, \log p^{\vec{\varepsilon}}\left(z_{0} \mid z_{-\infty}^{-1}\right)$ is the uniform limit of analytic functions and hence is analytic on $\Omega_{\mathbb{C}}$ (see [32, Theorem 2.4.1]).

For a given sequence $z=z_{-\infty}^{0}$, let $f(\vec{\varepsilon} ; z)=$ $\log p^{\vec{\varepsilon}}\left(z_{0} \mid z_{-\infty}^{-1}\right)$. Let $\left.D_{\vec{\varepsilon}} f\right|_{\overrightarrow{\varepsilon_{0}}}(z)$ denote the vector of partial derivatives of $f(\vec{\varepsilon} ; z)$ with respect to $\vec{\varepsilon}$ at $\vec{\varepsilon}=\overrightarrow{\varepsilon_{0}}$. Using (4.16) and the Cauchy integral formula in several variables [32] (which expresses the derivative of an analytic function at a point as an integral on a closed surface around the point), we obtain the following. There is a positive constant $C^{\prime}$ such that whenever $z \stackrel{n}{\sim} \hat{z}$, for all $\overrightarrow{\varepsilon_{0}} \in \Omega_{\mathbb{C}}$

$$
\left|D_{\vec{\varepsilon}} f\right|_{\overrightarrow{\varepsilon_{0}}}(z)-\left.D_{\vec{\varepsilon}} f\right|_{\overrightarrow{\varepsilon_{0}}}(\hat{z}) \mid \leq C^{\prime} \rho^{n} \text {. }
$$

For a direction $\vec{h}$ in the parameter space, let $\left.D_{\vec{\varepsilon}} f\right|_{\overrightarrow{\varepsilon_{0}}}(\vec{h} ; z)$ denote the directional derivative of $f(\vec{\varepsilon} ; z)$ at $\vec{\varepsilon}=\overrightarrow{\varepsilon_{0}}$ in direction $\vec{h}$. Let $\left.D_{\vec{\varepsilon}} f\right|_{\overrightarrow{\varepsilon_{0}}}(\vec{h} ; \cdot)$ denote the function on $\mathcal{X}$, whose value on $z=$ $z_{-\infty}^{0} \in \mathcal{X}$ is given by $\left.D_{\vec{\varepsilon}} f\right|_{\overrightarrow{\varepsilon_{0}}}(\vec{h} ; z)$. By $(8.32),\left.D_{\vec{\varepsilon}} f\right|_{\overrightarrow{\varepsilon_{0}}}(\vec{h} ; \cdot)$ belongs to $F_{\mathbb{C}}^{\rho}$. 
Now, we must prove that the mapping $\vec{\varepsilon} \mapsto \log p^{\vec{\varepsilon}}\left(z_{0} \mid z_{-\infty}^{-1}\right)$ is complex differentiable (therefore analytic) from $\Omega_{\mathbb{C}}$ to $F_{\mathbb{C}}^{\rho}$. For this, it suffices to prove that for all $\overrightarrow{\varepsilon_{0}} \in \Omega_{\mathbb{C}}$

$$
\left\|f\left(\overrightarrow{\varepsilon_{0}}+\vec{h} ; \cdot\right)-f\left(\overrightarrow{\varepsilon_{0}} ; \cdot\right)-\left.D_{\vec{\varepsilon}} f\right|_{\overrightarrow{\varepsilon_{0}}}(\vec{h} ; \cdot)\right\|_{\infty} \leq o(|\vec{h}|)
$$
and

$$
\left\|f\left(\overrightarrow{\varepsilon_{0}}+\vec{h} ; \cdot\right)-f\left(\overrightarrow{\varepsilon_{0}} ; \cdot\right)-\left.D_{\vec{\varepsilon}} f\right|_{\overrightarrow{\varepsilon_{0}}}(\vec{h} ; \cdot)\right\|_{\rho} \leq o(|\vec{h}|) .
$$

Let $\left.D_{\vec{\varepsilon}}^{2} f\right|_{\overrightarrow{\varepsilon_{0}}}(\vec{h}, \vec{h} ; z)$ denote the second directional derivative in direction $(\vec{h}, \vec{h})$ of $f(\vec{\varepsilon} ; z)$ at $\vec{\varepsilon}=\overrightarrow{\varepsilon_{0}}$. Again, applying the Cauchy integral formula in several variables, it follows that there exists a positive constant $C^{\prime \prime}$ such that for all $\overrightarrow{\varepsilon_{0}} \in \Omega_{\mathbb{C}}$ we have

$$
\left.\left|D_{\vec{\varepsilon}}^{2} f\right|_{\overrightarrow{\varepsilon_{0}}}(\vec{h}, \vec{h} ; z)\left|\leq C^{\prime \prime}\right| \vec{h}\right|^{2}
$$

and whenever $z \stackrel{n}{\sim} \hat{z}$

$$
\begin{array}{r}
\int_{0}^{1}(1-t)\left|\left(\left.D_{\vec{\varepsilon}}^{2} f\right|_{\overrightarrow{\varepsilon_{0}}}(\vec{h}, \vec{h} ; z)-\left.D_{\vec{\varepsilon}}^{2} f\right|_{\overrightarrow{\varepsilon_{0}}}(\vec{h}, \vec{h} ; \hat{z})\right)\right| d t \\
\leq C^{\prime \prime}|\vec{h}|^{2} \rho^{n} .
\end{array}
$$

From the Taylor formula with integral remainder, we have

$$
\begin{aligned}
f\left(\overrightarrow{\varepsilon_{0}}+\vec{h} ; z\right)-f\left(\overrightarrow{\varepsilon_{0}} ; z\right)-\left.D_{\vec{\varepsilon}} f\right|_{\overrightarrow{\varepsilon_{0}}}(\vec{h} ; z) \\
\quad=\left.\int_{0}^{1}(1-t) D_{\vec{\varepsilon}}^{2} f\right|_{\overrightarrow{\varepsilon_{0}}+t \vec{h}}(\vec{h}, \vec{h} ; z) d t .
\end{aligned}
$$

To prove (8.33), use (8.35) and (8.37). To prove (8.34), use (8.36) and (8.37). Therefore, $\vec{\varepsilon} \mapsto \log p^{\vec{\varepsilon}}(\cdot)$ is analytic as a mapping from $\Omega_{\mathbb{C}}$ to $F_{\mathbb{C}}^{\rho}$. Restricting the mapping $\vec{\varepsilon} \mapsto \log p^{\vec{\varepsilon}}\left(z_{0} \mid z_{-\infty}^{-1}\right)$ to the real parameter space, we conclude that it is real analytic (as a mapping into $F^{\rho}$ ). Using this and the theory of equilibrium states [26], the "Moreover" is proven in Appendix C.

Corollary 8.2: Suppose that at $\vec{\varepsilon}_{0}, \Delta$ satisfies Conditions 1 and 2 in Theorem 1.1, and $\vec{\varepsilon} \mapsto f^{\vec{\varepsilon}} \in F^{\rho}$ is analytic at $\vec{\varepsilon}_{0}$, then $\vec{\varepsilon} \mapsto \nu^{\vec{\varepsilon}}(f \vec{\varepsilon})$ is analytic at $\vec{\varepsilon}_{0}$. In particular, we recover Theorem 1.1: $\vec{\varepsilon} \mapsto H^{\vec{\varepsilon}}(Z)$ is analytic at $\vec{\varepsilon}_{0}$.

Proof: The map

$$
\begin{aligned}
& \Omega \rightarrow F^{\rho} \times\left(F^{\rho}\right)^{*} \rightarrow \mathbb{R} \\
& \vec{\varepsilon} \mapsto\left(f^{\vec{\varepsilon}}, \nu^{\vec{\varepsilon}}\right) \mapsto \nu^{\vec{\varepsilon}}\left(f^{\vec{\varepsilon}}\right)
\end{aligned}
$$

is analytic at $\vec{\varepsilon}_{0}$, as desired.

\section{APPENDIX A}

\section{PROOF OF PROPOSITION 2.1}

Proof: Without loss of generality, we assume $S$ is convex (otherwise, consider the convex hull of $S$ ). It follows from standard arguments that max and sum norms are equivalent. More specifically, for another metric $d_{1}$ defined by

$$
d_{1}(u, v)=\sqrt{\sum_{i \neq j \leq k} \log ^{2}\left(\frac{u_{i} / u_{j}}{v_{i} / v_{j}}\right)}
$$

we have $d_{\mathbf{B}} \sim d_{1}$. For metric $d_{2}$ defined by

$$
d_{2}(u, v)=\sqrt{\sum_{i \neq j \leq k}\left(u_{i} / u_{j}-v_{i} / v_{j}\right)^{2}} .
$$

Applying mean value theorem to $\log$ function, one concludes that $d_{1} \sim d_{2}$. Note that

$$
\begin{aligned}
u_{i}-v_{i}= & \frac{u_{i}}{u_{1}+u_{2}+\cdots+u_{k}} \\
& -\frac{v_{i}}{v_{1}+v_{2}+\cdots+v_{k}} \\
= & \frac{1}{u_{1} / u_{i}+u_{2} / u_{i}+\cdots+u_{k} / u_{i}} \\
& -\frac{1}{v_{1} / v_{i}+v_{2} / v_{i}+\cdots+v_{k} / v_{i}} .
\end{aligned}
$$

Applying the mean value theorem to function $f$, defined as

$$
f\left(x_{1}, x_{2}, \ldots, x_{B}\right)=\frac{1}{x_{1}+x_{2}+\cdots+x_{k}}
$$

we conclude that there exists $\xi \in S$ such that

$$
u_{i}-v_{i}=\left.\nabla f\right|_{\xi} \cdot\left(u_{1} / u_{i}-v_{1} / v_{i}, \ldots, u_{k} / u_{i}-v_{k} / v_{i}\right) .
$$

It follows from Cauchy inequality that there exists a positive constant $D_{1}$ such that

$$
d_{\mathbf{E}}(u, v)<D_{1} d_{2}(u, v) .
$$

Similarly, consider $u_{i} / u_{j}-v_{i} / v_{j}$, and apply mean value theorem to function $g$, defined as $g(x, y)=x / y$, we show that there exists a positive constant $D_{2}$ such that

$$
d_{2}(u, v)<D_{2} d_{\mathbf{E}}(u, v) .
$$

Namely, $d_{2} \sim d_{E}$. Thus, the claim in this proposition follows, namely, there exist two positive constant $C_{1}<C_{2}$ such that for any two points $u, v \in S$,

$$
C_{1} d_{\mathbf{B}}(u, v)<d_{\mathbf{E}}(u, v)<C_{2} d_{\mathbf{B}}(u, v) .
$$

\section{APPENDIX B}

PROOF OF LEMMA 7.9

Recall that for a nonnegative matrix $B$, the canonical form of $B$ is

$$
B=\left[\begin{array}{cccc}
B_{11} & B_{12} & \cdots & B_{1 n} \\
0 & B_{22} & \cdots & B_{2 n} \\
\vdots & \vdots & \ddots & \vdots \\
0 & 0 & \cdots & B_{n n}
\end{array}\right]
$$

where $B_{i i}$ is either an irreducible matrix (called irreducible components) or a $1 \times 1$ zero matrix.

Condition 2 in Theorem 7.8 is equivalent to the statement that $B=B\left(\vec{\varepsilon}_{0}\right)$ has a unique irreducible component of maximal 
spectral radius and that this component is primitive. Let $C$ denote the square matrix obtained by restricting $B$ to this component and let $S_{C}$ denote the set of indices corresponding to this component. Let $\lambda_{1}$ denote the spectral radius of $B$, equivalently, the spectral radius of $C$.

Let $\lambda_{1}(\vec{\varepsilon})$ denote the largest, in modulus, eigenvalue of $B(\vec{\varepsilon})$. Since the entries of $B(\vec{\varepsilon})$ are analytic in $\vec{\varepsilon}$ and $\lambda_{1}$ is simple, it follows that if the complex neighborhood $\Omega$ is chosen sufficiently small, then $\lambda_{1}(\vec{\varepsilon})$ is an analytic function of $\vec{\varepsilon} \in \Omega$.

The columns (resp., rows) of $\operatorname{Adj}\left(\lambda_{1}(\vec{\varepsilon}) I-B(\vec{\varepsilon})\right)$ are right (resp., left) eigenvectors of $B(\vec{\varepsilon})$ corresponding to $\lambda_{1}(\vec{\varepsilon})$. By choosing $x(\vec{\varepsilon})$ (resp., $y(\vec{\varepsilon})$ ) to be a fixed column (resp., row) of $\operatorname{Adj}\left(\lambda_{1}(\vec{\varepsilon}) I-B(\vec{\varepsilon})\right)$ and then replacing $x(\vec{\varepsilon})$ and $y(\vec{\varepsilon})$ by appropriately rescaled versions, we may assume that

- $x\left(\vec{\varepsilon}_{0}\right), y\left(\vec{\varepsilon}_{0}\right) \geq 0$, and they are positive on $S_{C}$;

- $y(\vec{\varepsilon}) \cdot x(\vec{\varepsilon})=1$

- $x(\vec{\varepsilon})$ and $y(\vec{\varepsilon})$ are analytic in $\vec{\varepsilon} \in \Omega$.

Let

$$
V(\vec{\varepsilon})=\lambda_{1}(\vec{\varepsilon}) x(\vec{\varepsilon}) \cdot y(\vec{\varepsilon})
$$

and

$$
U(\vec{\varepsilon})=B(\vec{\varepsilon})-V(\vec{\varepsilon})
$$

Then

$$
\begin{aligned}
U(\vec{\varepsilon}) V(\vec{\varepsilon}) & =B(\vec{\varepsilon}) V(\vec{\varepsilon})-V^{2}(\vec{\varepsilon}) \\
& =\lambda_{1}^{2}(\vec{\varepsilon}) x(\vec{\varepsilon}) \cdot y(\vec{\varepsilon})-\lambda_{1}^{2}(\vec{\varepsilon}) x(\vec{\varepsilon}) \cdot y(\vec{\varepsilon})=\mathbf{0} .
\end{aligned}
$$

And similarly

$$
V(\vec{\varepsilon}) U(\vec{\varepsilon})=\mathbf{0}
$$

Let $\mu(\vec{\varepsilon})$ denote the spectral radius of $U(\vec{\varepsilon})$. By Condition 2, $\mu\left(\vec{\varepsilon}_{0}\right)<\lambda_{1}\left(\vec{\varepsilon}_{0}\right)$. Thus, there is a constant $\nu>0$ such that if the neigbourhood $\Omega$ is sufficiently small, then for all $\vec{\varepsilon} \in \Omega$

$$
\mu(\vec{\varepsilon})<\nu<\left|\lambda_{1}(\vec{\varepsilon})\right| \text {. }
$$

Thus, by Lemma 7.5, and making still $\Omega$ smaller if necessary, there is a constant $K_{1}>0$ such that for all $i, j$, all $n$ and all $\vec{\varepsilon} \in \Omega$

$$
\left|U_{i j}^{n}(\vec{\varepsilon})\right|<K_{1} \nu^{n}
$$

Let $r=r\left(\vec{\varepsilon}_{0}\right), c=c\left(\vec{\varepsilon}_{0}\right), x=x\left(\vec{\varepsilon}_{0}\right)$ and $y=y\left(\vec{\varepsilon}_{0}\right)$.

Let $s_{0} \in S_{C}$. Since $\Delta\left(\vec{\varepsilon}_{0}\right)$ is irreducible, for some $j_{0},\left(r B^{j_{0}}\right)_{s_{0}}>0$. Similarly, there exist a state $s_{1}$ of the underlying Markov chain and $j_{1}$ such that $B_{s_{0} s_{1}}^{j_{1}} c_{s_{1}}>0$. Now

$$
r B^{n} c \geq\left(r B^{j_{0}}\right)_{s_{0}} C_{s_{0} s_{0}}^{n-j_{0}-j_{1}} B_{s_{0} s_{1}}^{j_{1}} c_{s_{1}} .
$$

Since $C$ is primitive, by Perron-Frobenius theory, $C_{s_{0} s_{0}}^{n-j_{0}-j_{1}}$ grows like $\lambda_{1}^{n-j_{0}-j_{1}}$ (up to a scalar) as $n$ goes to infinity; it then follows that there is a constant $K_{2}$ such that for sufficiently large $n$

$$
\begin{aligned}
r x \cdot y c \lambda_{1}^{n}+r U^{n} c & =r V^{n} c+r U^{n} c \\
& =r B^{n} c>K_{2} \lambda_{1}^{n}
\end{aligned}
$$

which by (B.38) implies that $r x \cdot y c>0$. Therefore, if $\Omega$ is sufficiently small, there exists a positive constant $K_{4}$ such that

$$
|r(\vec{\varepsilon}) x(\vec{\varepsilon}) \cdot y(\vec{\varepsilon}) c(\vec{\varepsilon})|>K_{4}
$$

for $\vec{\varepsilon} \in \Omega$.

Let $K_{3}$ be an upper bound on the entries of $|x(\vec{\varepsilon})|,|y(\vec{\varepsilon})|$, $|r(\vec{\varepsilon})|$, and $|c(\vec{\varepsilon})|$.

Thus, for all $n$ and all $\vec{\varepsilon} \in \Omega$, we have

$$
\begin{aligned}
\left|r(\vec{\varepsilon}) B^{n}(\vec{\varepsilon}) c(\vec{\varepsilon})\right| & \leq\left|r(\vec{\varepsilon}) U^{n}(\vec{\varepsilon}) c(\vec{\varepsilon})\right|+\left|r(\vec{\varepsilon}) V^{n}(\vec{\varepsilon}) c(\vec{\varepsilon})\right| \\
& \leq|\mathcal{B}|^{2} K_{3}^{2} K_{1} \nu^{n}+|\mathcal{B}|^{2} K_{3}^{4}\left|\lambda_{1}(\vec{\varepsilon})\right|^{n}
\end{aligned}
$$

and

$$
\begin{aligned}
\left|r(\vec{\varepsilon}) B^{n}(\vec{\varepsilon}) c(\vec{\varepsilon})\right| & \geq\left|r(\vec{\varepsilon}) V^{n}(\vec{\varepsilon}) c(\vec{\varepsilon})\right|-\left|r(\vec{\varepsilon}) U^{n}(\vec{\varepsilon}) c(\vec{\varepsilon})\right| \\
& \geq K_{4}\left|\lambda_{1}(\vec{\varepsilon})\right|^{n}-|\mathcal{B}|^{2} K_{3}^{2} K_{1} \nu^{n} .
\end{aligned}
$$

With similar upper and lower bounds for $\left|r(\vec{\varepsilon}) B^{n}(\vec{\varepsilon}) \mathbf{1}\right|$, it follows that for sufficiently large $n$ and all $\vec{\varepsilon} \in \Omega$,

and

$$
\frac{\pi_{1}(\vec{\varepsilon}) r(\vec{\varepsilon}) B(\vec{\varepsilon})^{n} \mathbf{1}}{\pi_{1}(\vec{\varepsilon}) r(\vec{\varepsilon}) B(\vec{\varepsilon})^{n-1} \mathbf{1}}
$$

$$
\frac{\pi_{1}(\vec{\varepsilon}) r(\vec{\varepsilon}) B(\vec{\varepsilon})^{n-1} c(\vec{\varepsilon})}{\pi_{1}(\vec{\varepsilon}) r(\vec{\varepsilon}) B(\vec{\varepsilon})^{n-1} \mathbf{1}}
$$

are uniformly bounded from above and away from zero. By Condition 1 , for any finite collection of $n$, there is a (possibly smaller) neighborhood $\Omega$ of $\vec{\varepsilon}_{0}$, such that for all $\vec{\varepsilon} \in \Omega$, these quantities are uniformly bounded from above and away from zero. This completes the proof of Lemma 7.9 (and therefore, the proof of sufficiency for Theorem 7.8).

\section{APPENDIX C \\ $\vec{\varepsilon} \mapsto \nu^{\vec{\varepsilon}}$ IS ANALYTIC}

In this appendix, we follow the notation in Section VIII. Let $\tau: \mathcal{X} \rightarrow \mathcal{X}$ be the right shift operator, which is a continuous mapping on $\mathcal{X}$ under the topology induced by the metric $d$. For $f \in C(\mathcal{X})$, one defines the pressure via a variational principle [26]

$$
P(f)=\sup _{\mu \in M(\mathcal{X}, \tau)}\left(H_{\mu}(\tau)+\int f d \mu\right)
$$

where $M(\mathcal{X}, \tau)$ denotes the set of $\tau$-invariant probability measures on $\mathcal{X}$ and $H_{\mu}(\tau)$ denotes measure-theoretic entropy. A member $\mu$ of $M(\mathcal{X}, \tau)$ is called an equilibrium state for $f$ if $P(f)=H_{\mu}(T)+\int f d \mu$.

For $f \in C(\mathcal{X})$, the Ruelle operator $\mathcal{L}_{f}: C(\mathcal{X}) \rightarrow C(\mathcal{X})$ is defined [26] by

$$
\left(\mathcal{L}_{f} h\right)(x)=\sum_{y \in \tau^{-1}(x)} e^{f(y)} h(y) .
$$

The connection between pressure and the Ruelle operator is as follows [26], [28]. When $f \in F^{\theta}, P(f)$ is $\log \lambda$, where $\lambda$ is the spectral radius of $\mathcal{L}_{f}$. The restriction of $\mathcal{L}_{f}$ to $F^{\theta}$ still has spectral radius $\lambda$, and $\lambda$ is isolated from all other eigenvalues of the restricted operator. Using this, Ruelle applied standard 
perturbation theory for linear operators [13] to conclude that pressure $P(f)$ is real analytic on $F^{\theta}$. Moreover, he showed that each $f \in F^{\theta}$ has a unique equilibrium state $\mu_{f}$ and the firstorder derivative of $f \mapsto P(f)$ on $F^{\theta}$ is $\mu_{f}$, viewed as a linear functional on $F^{\theta}$. So, the analyticity of $P(f)$ implies that the equilibrium state $\mu_{f}$ is also analytic in $f \in F^{\theta}$.

We first claim that for $f(\vec{\varepsilon}, z)=\log p^{\vec{\varepsilon}}\left(z_{0} \mid z_{-\infty}^{-1}\right)$, we have $\mu_{f(\vec{\varepsilon}, \cdot)}=\nu^{\vec{\varepsilon}}$ as in (8.30).

To see this, first observe that the spectral radius $\lambda$ of $\mathcal{L}=$ $\mathcal{L}_{f(\vec{\varepsilon}, \cdot)}$ is 1 ; this follows from the following observations:

- the function $\overline{1}$ which is identically 1 on $\mathcal{X}$ is a fixed point of $\mathcal{L}$ and

- (see [26, Proposition 5.16]) $\mathcal{L}^{n}(\overline{1}) / \lambda^{n}$ converges to a strictly positive function.

Thus, $P(f(\vec{\varepsilon}, \cdot))=0$. So, for $\mu^{\vec{\varepsilon}}=\mu_{f(\vec{\varepsilon}, \cdot)}$, we have

$$
h_{\mu^{\vec{\varepsilon}}}(\tau)+\int f(\vec{\varepsilon}, \cdot) d \mu^{\vec{\varepsilon}}=0 .
$$

But from (8.31), we have

$$
h_{\nu}(\tau)+\int f(\vec{\varepsilon}, \cdot) d \nu^{\vec{\varepsilon}}=0 .
$$

By uniqueness of the equilibrium state, we thus obtain $\mu_{f(\vec{\varepsilon}, \cdot)}=\nu^{\vec{\varepsilon}}$ as claimed.

Since $\vec{\varepsilon} \mapsto f(\vec{\varepsilon}, \cdot)$ is analytic, it then follows that $\vec{\varepsilon} \mapsto \nu^{\vec{\varepsilon}}$ is analytic, thereby completing the proof of Theorem 8.1.

\section{ACKNOWLEDGMENT}

The authors are grateful to Wael Bahsoun, Joel Feldman, Robert Israel, Izabella Laba, Erik Ordentlich, Yuval Peres, Gadiel Seroussi, Wojciech Szpankowski, and Tsachy Weissman for helpful discussions.

\section{REFERENCES}

[1] L. Arnold, V. M. Gundlach, and L. Demetrius, "Evolutionary formalism for products of positive random matrices," Ann. Appl. Probab., vol. 4, pp. 859-901, 1994.

[2] D. Arnold and H. Loeliger, "The information rate of binary-input channels with memory," in Proc. 2001 IEEE Int. Conf. Communications, Helsinki, Finland, Jun. 2001, pp. 2692-2695.

[3] J. J. Birch, "Approximations for the entropy for functions of Markov chains," Ann. Math. Statist., vol. 33, pp. 930-938, 1962.

[4] D. Blackwell, "The entropy of functions of finite-state Markov chains," in Trans. First Prague Conf. Information Theory, Statistical Decision Functions, Random Processes, Prague, Czechoslovakia (now Czech Republic), 1957, pp. 13-20.

[5] M. Cassandro and E. Olivieri, "Renormalization group and analyticity in one dimension: A proof of Dobrushin's theorem," Commun. Math. Phys., vol. 80, pp. 255-269, 1981.

[6] J. R. Chazottes and E. Ugalde, "Projection of Markov measures may be Gibbsian," J. Statist. Phys., vol. 111, no. 5-6, pp. 1245-1272, Jun. 2003.

[7] R. L. Dobrushin, "Analyticity of correlation functions in one-dimensional classical systems with slowly decreasing potentials," Commun. Math. Phys., vol. 32, pp. 269-289, 1973.

[8] S. Egner, V. Balakirsky, L. Tolhuizen, S. Baggen, and H. Hollmann, "On the entropy rate of a hidden Markov model," in Proc. IEEE Int. Symp. Information Theory, Chicago, IL, Jun.Jul. 2004, p. 12.

[9] G. Han and B. Marcus, "Analyticity of entropy rate of a hidden Markov chain," in Proc. IEEE Int. Symp. Information Theory, Adelaide, Australia, Sep. 2005, pp. 2193-2197.
[10] R. Gharavi and V. Anantharam, An Upper Bound for the Largest Lyapunov Exponent of a Markovian Product of Nonnegative Matrices, Jan. 1995, Preprint.

[11] T. Holliday, A. Goldsmith, and P. Glynn, On Entropy and Lyapunov Exponents for Finite State Channels 2003 [Online]. Available: http:// wsl.stanford.edu/Publications/THolliday/Lyapunov.pdf

[12] P. Jacquet, G. Seroussi, and W. Szpankowski, "On the entropy of a hidden Markov process," in Proc. IEEE Int. Symp. Information Theory, Chicago, IL, Jun./Jul. 2004, p. 10.

[13] T. Kato, Perturbation Theory for Linear Operators. Berlin-Heidelberg-New York: Springer-Verlag, 1976.

[14] D. Lind and B. Marcus, An Introduction to Symbolic Dynamics and Coding. Cambridge, U.K.: Cambridge Univ. Press, 1995.

[15] J. Lörinczi, C. Maes, and K. V. Velde, "Transformations of Gibbs measures," Probab. Theory Relat. Fields, vol. 112, pp. 121-147, 1998.

[16] B. Marcus, K. Petersen, and S. Williams, "Transmission rates and factors of Markov chains," Contemp. Math., vol. 26, pp. 279-294, 1984.

[17] A. Mukherjea and K. Pothoven, Real and Functional Analysis. New York: Plenum, 1978.

[18] L. Nachbin, Introduction to Functional Analysis : Banach Spaces and Differential Calculus. New York: Marcel Dekker, 1981.

[19] A. Onishchik, Lie Groups and Lie Algebra I. New York: SpringerVerlag, 1993, Encyclopaedia of Mathematical Sciences; vol. 20.

[20] E. Ordentlich and T. Weissman, "On the optimality of symbol by symbol filtering and denoising," IEEE Trans. Inf. Theory, , vol. 52, no. 1, pp. 19-40, Jan. 2006.

[21] E. Ordentlich and T. Weissman, "New bounds on the entropy rate of hidden Markov process," in Proc. Information Theory Workshop, San Antonio, TX, Oct. 2004, pp. 117-122.

[22] Y. Peres, "Analytic dependence of Lyapunov exponents on transition probabilities," in Lecture Notes in Mathematics, Lyapunov's Exponents, Proceedings of a Workshop. Berlin, Germany: Springer-Verlag, 1991, vol. 1486, pp. 64-80.

[23] Y. Peres, "Domains of analytic continuation for the top Lyapunov exponent," Ann. Inst. H. Poincaré Probab. Statist., vol. 28, no. 1, pp. 131-148, 1992.

[24] K. Petersen, A. Quas, and S. Shin, "Measures of maximal relative entropy," in Ergod. Theorems and Dynam. Systems, 2003, vol. 23, pp. 207-223.

[25] H. Pfister, J. Soriaga, and P. Siegel, "The achievable information rates of finite-state ISI channels," in Proc. IEEE GLOBECOM, San Antonio, TX, Nov. 2001, pp. 2992-2996.

[26] D. Ruelle, Thermodynamic Formalism : The Mathematical Structures of Classical Equilibrium Statistical Mechanics. Reading, MA: Addison-Wesley-Advanced Book Program, 1978.

[27] D. Ruelle, "Analyticity properties of the characteristic exponents of random matrix products," Adv. Math., vol. 32, pp. 68-80, 1979.

[28] D. Ruelle, "Differentiation of SRB states," Commun. Math. Phys., vol. 187, no. 1, pp. 227-241, 1997.

[29] E. Seneta, Non-negative Matrices and Markov Chains. New YorkHeidelberg-Berlin: Springer-Verlag, 1980, Springer Series in Statistics.

[30] B. V. Shabat, Introduction to Complex Analysis, ser. Translations of Mathematical Monographs. Providence, RI: Amer. Math. Soc., 1992, vol. 110.

[31] V. Sharma and S. Singh, "Entropy and channel capacity in the regenerative setup with applications to Markov channels," in Proc. IEEE Int. Symp. Information Theory, Washington, DC, Jun. 2001, p. 283.

[32] J. L. Taylor, Several Complex Variables with Connections to Algebraic Geometry and Lie Groups. Providence, RI: Amer. Math. Soc., 2002.

[33] P. Walters, An Introduction to Ergodic Theory, ser. Graduate Texts in Mathematics. New York: Springer-Verlag, 1982, vol. 79.

[34] K. Yosida, Functional Analysis, 4th ed. Berlin, Germany: SpringerVerlag, 1974.

[35] O. Zuk, I. Kanter, and E. Domany, "The entropy of a binary hidden Markov process," J. Stat ist. Phys., vol. 121, no. 3-4, pp. 343-360, 2005.

[36] O. Zuk, E. Domany, I. Kanter, and M. Aizenman, "Taylor series expansions for the entropy rate of hidden Markov Processes," in Proc. Int. Conf. Communications, Istanbul, Turkey, Jun. 2006. 